\title{
Generalizations of Cesàro means and poles of the resolvent
}

\author{
by \\ Laura Burlando (Genova)
}

\begin{abstract}
An improvement of the generalization-obtained in a previous article [Bu1] by the author - of the uniform ergodic theorem to poles of arbitrary order is derived. In order to answer two natural questions suggested by this result, two examples are also given. Namely, two bounded linear operators $T$ and $A$ are constructed such that $n^{-2} T^{n}$ converges uniformly to zero, the sum of the range and the kernel of $1-T$ being closed, and $n^{-3} \sum_{k=0}^{n-1} A^{k}$ converges uniformly, the sum of the range of $1-A$ and the kernel of $(1-A)^{2}$ being closed. Nevertheless, 1 is a pole of the resolvent of neither $T$ nor $A$.
\end{abstract}

1. Introduction. Throughout this paper, when the scalar field is not specified, we assume it may be either $\mathbb{R}$ or $\mathbb{C}$ and denote it by $\mathbb{K}$. Also, we denote the norm of any normed space $\mathcal{X}$ by \|\|$_{\mathcal{X}}$.

For each Banach space $X$, let $0_{X}, L(X)$ and $I_{X}$ denote respectively the zero element of $X$, the Banach algebra of all bounded linear operators on $X$ and the identity element of $L(X)$. For each $T \in L(X)$, let $\mathcal{N}(T)$ and $\mathcal{R}(T)$ denote the kernel and the range of $T$, respectively. Furthermore, if $T$ is invertible in $L(X)$, we denote the inverse of $T$ in $L(X)$ by $T^{-1}$. A projection of $X$ is an element $P$ of $L(X)$ satisfying $P^{2}=P$. We recall that if $P$ is a projection of $X$, then $\mathcal{R}(P)$ is closed and $X=\mathcal{R}(P) \oplus \mathcal{N}(P)$. Conversely, if $Y$ and $Z$ are closed subspaces of $X$ satisfying $X=Y \oplus Z$, then there exists a unique projection $P$ of $X$-called the projection of $X$ onto $Y$ along $Z$ - such that $\mathcal{R}(P)=Y$ and $\mathcal{N}(P)=Z$.

Now suppose $X$ to be a complex Banach space. For each $T \in L(X)$, let $\sigma(T)$ and $\varrho(T)$ stand respectively for the spectrum of $T$ and the resolvent set $\mathbb{C} \backslash \sigma(T)$ of $T$. As is well known, the resolvent function

$$
R(\cdot, T): \varrho(T) \ni \lambda \mapsto\left(\lambda I_{X}-T\right)^{-1} \in L(X)
$$

is holomorphic on $\varrho(T)$. We set

$$
\mathcal{P}(T)=\varrho(T) \cup\{\lambda \in \sigma(T): \lambda \text { is a pole of } R(\cdot, T)\} .
$$

2000 Mathematics Subject Classification: Primary 47A35, 47A10.

Key words and phrases: uniform ergodic theorem, poles of the resolvent, ranges and kernels of the iterates. 
Throughout this paper, $\mathbb{N}$ and $\mathbb{Z}_{+}$will stand for the sets of all nonnegative integers and of all positive integers, respectively. For each $p \in \mathbb{Z}_{+}$, we set

$$
\mathcal{P}_{p}(T)=\varrho(T) \cup\{\lambda \in \sigma(T): \lambda \text { is a pole of } R(\cdot, T) \text { of order } \leq p\} .
$$

We also set $\mathcal{P}_{0}(T)=\varrho(T)$. We recall that for each $\lambda_{0} \in \mathcal{P}_{p}(T)$ (where $p \in \mathbb{N}$ ) we have $X=\mathcal{N}\left(\left(\lambda_{0} I_{X}-T\right)^{p}\right) \oplus \mathcal{R}\left(\left(\lambda_{0} I_{X}-T\right)^{p}\right)$ and $\mathcal{R}\left(\left(\lambda_{0} I_{X}-T\right)^{p}\right)$ is closed (see, for instance, Theorem 2.2 in the next section). Notice also that, for each $p \in \mathbb{N}, \mathcal{P}_{p}(T)$ is an open subset of $\mathbb{C}$ and $\mathcal{P}_{p}(T) \subset \mathcal{P}_{p+1}(T)$. Finally, we remark that $\mathcal{P}(T)=\bigcup_{p \in \mathbb{N}} \mathcal{P}_{p}(T)$.

We are concerned here with conditions - related to convergence in $L(X)$ of a convenient generalization of the Cesàro means of the sequence of the iterates of $T$-ensuring that $1 \in \mathcal{P}_{p}(T)$.

The classical uniform ergodic theorem by N. Dunford ([D1], [D2]) shows that $1 \in \mathcal{P}(T)$ and $n^{-1}\left\|T^{n}\right\|_{L(X)} \rightarrow 0$ as $n \rightarrow \infty$ if and only if the sequence $n^{-1} \sum_{k=0}^{n-1} T^{k}$ converges in $L(X)$; moreover, in this case, $1 \in \mathcal{P}_{1}(T)$ and $n^{-1} \sum_{k=0}^{n-1} T^{k}$ converges to the projection of $X$ onto $\mathcal{N}\left(I_{X}-T\right)$ along $\mathcal{R}\left(I_{X}-T\right)$ (which coincides with the residue of $R(\cdot, T)$ at 1 ; see [TL, V, (10-1) and 10.1]). Further conditions equivalent to the convergence of $n^{-1} \sum_{k=0}^{n-1} T^{k}$ in $L(X)$, improving Dunford's uniform ergodic theorem, have been obtained more recently by several authors (see [Li], [MZ], [LM]). A partial generalization of the uniform ergodic theorem to poles of arbitrary order was provided in $[\mathrm{W}]$ by H.-D. Wacker, who proved that if $p \in \mathbb{Z}_{+}$, $1 \in \mathcal{P}_{p}(T)$ and $n^{-p}\left\|T^{n}\right\|_{L(X)} \rightarrow 0$ as $n \rightarrow \infty$, then $n^{-p} \sum_{k=0}^{n-1} T^{k}$ converges in $L(X)$ to $(1 / p !)\left(T-I_{X}\right)^{p-1} P$ (where $P$ denotes the projection of $X$ onto $\mathcal{N}\left(\left(I_{X}-T\right)^{p}\right)$ along $\mathcal{R}\left(\left(I_{X}-T\right)^{p}\right)$; notice that $\left(T-I_{X}\right)^{p-1} P$ is the coefficient of order $-p$ of the Laurent expansion of $R(\cdot, T)$ in a punctured neighborhood of 1 by [TL, V, (10-1), (10-7) and 10.1]). In [W] an example is also constructed showing that convergence of $n^{-p} \sum_{k=0}^{n-1} T^{k}$ in $L(X)$ is not sufficient for $1 \in \mathcal{P}(T)$. In [Bu1] we obtained a converse of Wacker's result, by proving that convergence of $n^{-p} \sum_{k=0}^{n-1} T^{k}$ in $L(X)$, together with an additional condition which is automatically satisfied for $p=1$ (namely, closedness of $\mathcal{R}\left(\left(I_{X}-T\right)^{p-1}\right)+\mathcal{N}\left(I_{X}-T\right)$ ), is indeed equivalent to membership of 1 in $\mathcal{P}(T)$ plus convergence of $n^{-p}\left\|T^{n}\right\|_{L(X)}$ to zero. The main result of $[\mathrm{Bu} 1]([\mathrm{Bu} 1,3.4])$ actually provides several conditions that are equivalent to the two conditions above, thus generalizing the uniform ergodic theorem, as well as its improvements obtained in [Li], [MZ] and [LM], to poles of arbitrary order.

We are going to recall all of these conditions here, in Theorem 2.8. In this paper we mainly focus on the condition recorded here as (2.8.9), that is, convergence of $n^{-p}\left\|T^{n}\right\|_{L(X)}$ to zero, plus closedness of $\mathcal{R}\left(\left(I_{X}-T\right)^{k}\right)+$ 
$\mathcal{N}\left(\left(I_{X}-T\right)^{j}\right)$ for some $(k, j) \in \mathbb{N} \times \mathbb{N}$ satisfying $k \geq p$. Indeed, we are interested in determining whether and how $k<p$ can be allowed in (2.8.9), maintaining equivalence with the conditions of [Bu1, 3.4].

In Section 2 we collect some preliminaries, to make this paper as selfcontained as possible. We begin Section 3 with a real Banach space version of our generalization of the uniform ergodic theorem (Theorem 3.1). We also derive a further condition equivalent to the ones provided in [Bu1, 3.4] from a recent result obtained by S. Grabiner and J. Zemánek in [GZ]: more precisely, we observe that $k<p$ can be allowed in (2.8.9), provided $k \in \mathbb{Z}_{+}$and $k+j>p$ (Theorem 3.4). Furthermore, we construct an example showing that $1 \leq k<p$ cannot be allowed in (2.8.9) for $k+j=p$ (Example 3.6). Finally, by means of a convenient example (Example 3.10), we prove that closedness of $\mathcal{R}\left(\left(I_{X}-T\right)^{p-2}\right)+\mathcal{N}\left(\left(I_{X}-T\right)^{2}\right)$, plus convergence of $n^{-p} \sum_{k=0}^{n-1} T^{k}$ in $L(X)$, does not imply the equivalent conditions of [Bu1,3.4] even if $p \geq 3$ (that is, even if $p-2 \in \mathbb{Z}_{+}$).

2. Preliminaries. For every bounded linear operator $T$ on a Banach space $X$, let $\alpha(T)$ and $\delta(T)$ denote respectively the ascent and descent of $T$, that is,

$$
\begin{aligned}
& \alpha(T)=\inf \left\{n \in \mathbb{N}: \mathcal{N}\left(T^{n}\right)=\mathcal{N}\left(T^{n+1}\right)\right\}, \\
& \delta(T)=\inf \left\{n \in \mathbb{N}: \mathcal{R}\left(T^{n}\right)=\mathcal{R}\left(T^{n+1}\right)\right\} .
\end{aligned}
$$

Notice that $\alpha(T), \delta(T) \in \mathbb{N} \cup\{\infty\}$, and that $\alpha(T) \in \mathbb{N}$ (respectively, $\delta(T)$ $\in \mathbb{N}$ ) if and only if $\mathcal{N}\left(T^{n}\right)=\mathcal{N}\left(T^{n+1}\right)$ (respectively, $\mathcal{R}\left(T^{n}\right)=\mathcal{R}\left(T^{n+1}\right)$ ) for some $n \in \mathbb{N}$. Moreover, in this case, we have $\mathcal{N}\left(T^{k}\right)=\mathcal{N}\left(T^{\alpha(T)}\right)$ (respectively, $\mathcal{R}\left(T^{k}\right)=\mathcal{R}\left(T^{\delta(T)}\right)$ ) for all $k \in \mathbb{N}$ satisfying $k \geq \alpha(T)$ (respectively, $k \geq \delta(T)$ ). We also remark that $\alpha(T)=0$ (respectively, $\delta(T)=0$ ) if and only if $T$ is one-to-one (respectively, onto).

Finiteness of the ascent and descent of $T$ is related to a decomposition of $X$ into a direct sum of two closed subspaces (the range and kernel of a convenient iterate of $T$ ) by the following result.

Theorem 2.1 (see [TL, IV, 5.10 and V, 6.2-6.4]). Let $X$ be a Banach space and $T \in L(X)$.

If $\alpha(T)$ and $\delta(T)$ are finite, then $\alpha(T)=\delta(T), \mathcal{R}\left(T^{p}\right)$-where $p$ denotes the common value of $\alpha(T)$ and $\delta(T)$-is closed, and $X=\mathcal{R}\left(T^{p}\right) \oplus \mathcal{N}\left(T^{p}\right)$.

$$
\text { If } X=\mathcal{R}\left(T^{q}\right) \oplus \mathcal{N}\left(T^{q}\right) \text { for some } q \in \mathbb{Z}_{+} \text {, then } \alpha(T)=\delta(T) \leq q \text {. }
$$

We recall (see [TL, V.10]) that if $T$ is a bounded linear operator on a complex Banach space $X$ and $\lambda_{0} \in \mathcal{P}(T)$, then the residue of $R(\cdot, T)$ at $\lambda_{0}$ is a projection $P$ of $X$; furthermore, $P \neq 0_{L(X)}$ if and only if $\lambda_{0} \in \sigma(T)$, that 
is, if and only if $\lambda_{0}$ is a pole of $R(\cdot, T) . P$ is called the spectral projection of $T$ associated with $\lambda_{0}$.

The following classical result provides a characterization of membership of a complex number $\lambda_{0}$ in $\mathcal{P}(T)$, as well as a characterization of the spectral projection of $T$ associated with $\lambda_{0}$ in this case.

Theorem 2.2 (see [TL, V, 10.1 and 10.2]). Let $X$ be a complex Banach space, $T \in L(X)$ and $\lambda_{0} \in \mathbb{C}$. Then $\lambda_{0} \in \mathcal{P}(T)$ if and only if both $\alpha\left(\lambda_{0} I_{X}-T\right)$ and $\delta\left(\lambda_{0} I_{X}-T\right)$ are finite. Moreover, in this case, if we set $p=\min \left\{k \in \mathbb{N}: \lambda_{0} \in \mathcal{P}_{k}(T)\right\}$, we have $\alpha\left(\lambda_{0} I_{X}-T\right)=\delta\left(\lambda_{0} I_{X}-T\right)=p$ (from which, by Theorem 2.1 , we conclude that $\mathcal{R}\left(\left(\lambda_{0} I_{X}-T\right)^{p}\right)$ is closed and $\left.X=\mathcal{N}\left(\left(\lambda_{0} I_{X}-T\right)^{p}\right) \oplus \mathcal{R}\left(\left(\lambda_{0} I_{X}-T\right)^{p}\right)\right)$ and the spectral projection of $T$ associated with $\lambda_{0}$ coincides with the projection of $X$ onto $\mathcal{N}\left(\left(\lambda_{0} I_{X}-T\right)^{p}\right)$ along $\mathcal{R}\left(\left(\lambda_{0} I_{X}-T\right)^{p}\right)$.

In $[\mathrm{LM}], \mathrm{K}$. B. Laursen and M. Mbekhta introduced the following condition $(\mathcal{E}-k)$ (where $k \in \mathbb{Z}_{+}$) for a bounded linear operator $T$ on a Banach space $X$ (see [LM, Definition 2]): $T$ is said to satisfy condition $(\mathcal{E}-k)$ if

$$
\left\|\frac{1}{n}\left(I_{X}-T\right)^{k} \sum_{j=0}^{n-1} T^{j}\right\|_{L(X)} \rightarrow 0 \quad \text { as } n \rightarrow \infty .
$$

Clearly, since

$$
\left(I_{X}-T\right) \sum_{j=0}^{n-1} T^{j}=I_{X}-T^{n} \quad \text { for all } n \in \mathbb{Z}_{+},
$$

it follows that $T$ satisfies $(\mathcal{E}-k)$ if and only if $\left\|n^{-1}\left(I_{X}-T\right)^{k-1} T^{n}\right\|_{L(X)} \rightarrow 0$ as $n \rightarrow \infty$ (see also [LM, Lemma 3] and [Bu1, comments on p. 79]). We also recall that if $T$ satisfies $(\mathcal{E}$ - $k)$, then $\alpha\left(I_{X}-T\right) \leq k$ (see [LM, Proposition 4]). Condition $(\mathcal{E}-k)$ is involved in the characterizations of convergence of the sequence $n^{-1} \sum_{j=0}^{n-1} T^{j}$ in $L(X)$ which are given in [LM, Theorem 9]. Furthermore, in [LM, Theorem 6], several characterizations of membership of 1 in $P_{k}(T)$ are provided for $T$ satisfying $(\mathcal{E}-k)$.

Condition $(\mathcal{E}-k)$ is generalized in [Bu1] as follows (see [Bu1, 2.1]). Let $X$ be a Banach space, $T \in L(X)$, and $k, p \in \mathbb{Z}_{+}$. Then $T$ is said to satisfy condition $\mathcal{E}(k, p)$ if

$$
\left\|\frac{1}{n^{p}}\left(I_{X}-T\right)^{k} \sum_{j=0}^{n-1} T^{j}\right\|_{L(X)} \rightarrow 0 \quad \text { as } n \rightarrow \infty .
$$

If norm convergence is replaced by strong convergence in (2.4), then $T$ is said to satisfy condition $\mathcal{S}(k, p)$. Clearly, $\mathcal{E}(k, p)$ implies $\mathcal{S}(k, p)$. Also, $\mathcal{E}(k, p)$ (respectively, $\mathcal{S}(k, p)$ ) implies $\mathcal{E}(k+1, p)$ (respectively, $\mathcal{S}(k+1, p)$ ). From (2.3) (see also [Bu1, 2.2]) it follows that 
$T$ satisfies condition $\mathcal{E}(k, p)$ (respectively, $\mathcal{S}(k, p)$ ) if and only if

$$
\begin{aligned}
& \left\|\frac{1}{n^{p}}\left(I_{X}-T\right)^{k-1} T^{n}\right\|_{L(X)} \rightarrow 0 \text { as } n \rightarrow \infty \text { (respectively }, \\
& \left.\left\|\frac{1}{n^{p}}\left(I_{X}-T\right)^{k-1} T^{n} x\right\|_{X} \rightarrow 0 \text { as } n \rightarrow \infty \text { for all } x \in X\right) .
\end{aligned}
$$

Notice that $\mathcal{E}(k, 1)$ coincides with $(\mathcal{E}$ - $k)$ for every $k \in \mathbb{Z}_{+}$. Also, for each $p \in \mathbb{Z}_{+}$, from $(2.5)$ it follows that $T$ satisfies $\mathcal{E}(1, p)$ (respectively, $\mathcal{S}(1, p)$ ) if and only if $n^{-p}\left\|T^{n}\right\|_{L(X)} \rightarrow 0$ as $n \rightarrow \infty$ (respectively, $n^{-p}\left\|T^{n} x\right\|_{X} \rightarrow 0$ as $n \rightarrow \infty$ for all $x \in X)$.

The following result establishes a link between conditions $\mathcal{E}(k, p)$ (respectively, $\mathcal{S}(k, p))$ and $\mathcal{E}(k-j, p+j)$ (respectively, $\mathcal{S}(k-j, p+j)), j=0, \ldots, k-1$.

Theorem 2.6 ([Bu1, 2.4]). Let $X$ be a Banach space, $T \in L(X)$ and $k, p \in \mathbb{Z}_{+}$. If $T$ satisfies condition $\mathcal{E}(k, p)$ (respectively, $\mathcal{S}(k, p)$ ), then $T$ satisfies $\mathcal{E}(k-j, p+j)$ (respectively, $\mathcal{S}(k-j, p+j)$ ) for all $j=0, \ldots, k-1$. This, for $j=k-1$, gives $n^{-p-k+1}\left\|T^{n}\right\|_{L(X)} \rightarrow 0$ as $n \rightarrow \infty$ (respectively, $n^{-p-k+1}\left\|T^{n} x\right\|_{X} \rightarrow 0$ as $n \rightarrow \infty$ for all $\left.x \in X\right)$.

No converse of Theorem 2.6 holds. Indeed, a convenient example ([Bu1, 2.6]) shows that, for each integer $p \geq 2$, there exists an operator $T_{p}$ which satisfies $\mathcal{E}(1, p)$ and fails to satisfy $\mathcal{S}(2, p-1)$ (this, by Theorem 2.6, implies that $\mathcal{S}(k+1, p-k)$-and, a fortiori, $\mathcal{E}(k+1, p-k)$-is satisfied by $T_{p}$ for no $k=1, \ldots, p-1$; in particular, $T_{p}$ does not satisfy $(\mathcal{E}-p)$, which therefore turns out to be more restrictive than $\mathcal{E}(1, p)$ ).

Now let $X$ be a Banach space and $T \in L(X)$. It is known that strong convergence to zero of $n^{-q} T^{n}$ as $n \rightarrow \infty$ (where $q \in \mathbb{Z}_{+}$) implies $\alpha\left(I_{X}-T\right) \leq q$ (see [W, Satz 2]). Hence from Theorem 2.6 we get the following result (see also [Bu1, 2.5]).

$$
\begin{aligned}
& \text { If } T \text { satisfies condition } \mathcal{S}(k, p) \text { for some } k, p \in \mathbb{Z}_{+}, \text {then } \alpha\left(I_{X}-T\right) \leq \\
& p+k-1 \text {. }
\end{aligned}
$$

We call a bounded linear operator $A$ on a Banach space $X$ Kato decomposable if there exist two closed $A$-invariant subspaces $M$ and $N$ of $X$ such that $X=N \oplus M$, the operator $N \ni x \mapsto A x \in N$ is nilpotent, $A(M)$ is closed in $X$ and $A(M) \supset \mathcal{N}\left(A^{n}\right) \cap M$ for all $n \in \mathbb{N}$. As noted in [MO, Example (1) on p. 245], from the Kato decomposition theorem ([K, Theorem 4]) it follows that every bounded linear semi-Fredholm operator on $X$ is Kato decomposable.

We recall that the Kato decomposable operators have been introduced in [MO, Definition 3.1], where they are called quasi-Fredholm. Besides, the quasi-Fredholm operators had been previously introduced by J. P. Labrousse in the Hilbert space setting ([Lab, Definition 3.1.2]). For a bounded linear 
operator on a Hilbert space, the definition given by J. P. Labrousse is equivalent to Definition 3.1 of [MO] (see [Lab, Definition 3.2.1, Theorem 3.2.1 $\left({ }^{1}\right.$ ) and Theorem 3.2.2]; the Hilbert space is understood to be complex in [Lab], but the proofs of [Lab, Theorems 3.2.1 and 3.2.2] can be repeated without changes in the real Hilbert space case). We also recall that another definition of quasi-Fredholm operator on a Banach space, different from [MO, Definition 3.1], is given in [MM] and [KMMP]. In the special case of a bounded linear operator on a Hilbert space, this second definition is also equivalent to Definition 3.1.2 of [Lab]. Consequently, it is equivalent to Definition 3.1 of $[\mathrm{MO}]$ in the Hilbert space setting.

In [Bu1] we have followed the terminology of [MO, Definition 3.1], using the term "quasi-Fredholm" for Kato decomposable operators. Indeed, the authors of $[\mathrm{LM}]$, in a result that is improved by [Bu1, 3.4], had done the same (see [LM, Definition 5 and Theorem 6]). Nevertheless, we prefer here to adopt the term "Kato decomposable", in order to avoid misunderstanding, as it is the definition of quasi-Fredholm operator provided in $[\mathrm{MM}]$ and $[\mathrm{KMMP}]$, rather than the one given in $[\mathrm{MO}]$, that seems to be mostly used in recent literature $([\mathrm{P}],[\mathrm{Be}])$.

We are now ready to recall the main result of $[\mathrm{Bu} 1]$.

Theorem 2.8 ([Bu1, 3.4]). Let $p \in \mathbb{Z}_{+}, X$ be a complex Banach space and $T \in L(X)$. Then the following conditions are equivalent:

$$
\begin{aligned}
& n^{-p} \sum_{k=0}^{n-1} T^{k} \text { converges in } L(X) \text { and } \mathcal{R}\left(\left(I_{X}-T\right)^{p-1}\right)+\mathcal{N}\left(I_{X}-T\right) \\
& \text { is closed; } \\
& n^{-p}\left\|T^{n}\right\|_{L(X)} \rightarrow 0 \text { as } n \rightarrow \infty \text { and } 1 \in \mathcal{P}(T) ; \\
& T \text { satisfies condition } \mathcal{E}(k, p) \text { for some } k \in \mathbb{Z}_{+} \text {and } 1 \in \mathcal{P}_{p}(T) ;
\end{aligned}
$$

$\left({ }^{1}\right)$ We remark that the condition " $A(N) \subset N$ " in [Lab, Theorem 3.2.1, b)] is not a consequence of the two conditions " $A(\mathcal{D}(A) \cap M) \subset M$ " and " $N \subset \mathcal{N}\left(A^{d}\right)$ ", even for a quasi-Fredholm operator of degree $d$ and a decomposition of the Hilbert space satisfying also a) and c) of [Lab, Theorem 3.2.1]. Indeed, if $A$ is the bounded linear operator on the Hilbert space $\mathbb{K}^{3} \times \ell_{2}$, defined by $A\left(\left(\lambda_{0}, \lambda_{1}, \lambda_{2}\right), x\right)=\left(\left(0,0, \lambda_{1}\right), S x+\lambda_{0} e_{0}\right)$ for each $\left(\left(\lambda_{0}, \lambda_{1}, \lambda_{2}\right), x\right) \in \mathbb{K}^{3} \times \ell_{2}$ (where $S$ and $e_{0}$ denote respectively the backward shift operator on $\ell_{2}$ and the vector of the canonical basis of $\ell_{2}$ which spans $\mathcal{N}(S)$ ), it is not difficult to verify that $A$ is a quasi-Fredholm operator of degree 2 according to Definition 3.1.2 of [Lab]. Moreover, if we set $N=\mathbb{K}^{3} \times\left\{0_{\ell_{2}}\right\}$ and $M=\{(0,0,0)\} \times \ell_{2}$, it follows that $M$ and $N$ are closed subspaces of $\mathbb{K}^{3} \times \ell_{2}, \mathbb{K}^{3} \times \ell_{2}=M \oplus N, N \subset \mathcal{N}\left(A^{2}\right)$ and $A(M)=M$ (in particular, $M$ is $A$-invariant, $A(M)$ is closed and contains $\mathcal{N}\left(A^{n}\right) \cap M$ for all $\left.n \in \mathbb{N}\right)$. Nevertheless, $N$ is not $A$-invariant, as for instance $\left((1,0,0), 0_{\ell_{2}}\right) \in N$ and $A\left((1,0,0), 0_{\ell_{2}}\right)=$ $\left((0,0,0), e_{0}\right) \notin N$. Therefore the condition " $A(N) \subset N$ " should be explicitly required in

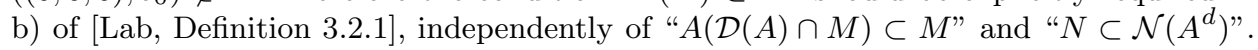
We also point out that the subspace $N$ constructed in the proof of [Lab, Theorem 3.2.1] is actually $A$-invariant. 
(2.8.4) T satisfies condition $\mathcal{E}(k, p)$ for some $k \in \mathbb{Z}_{+}$and

(2.8.5) $\quad n^{-p}\left\|T^{n}\right\|_{L(X)} \rightarrow 0$ as $n \rightarrow \infty$ and $\delta\left(I_{X}-T\right)<\infty$;

(2.8.6) $T$ satisfies condition $\mathcal{E}(k, p)$ for some $k \in \mathbb{Z}_{+}$and $\delta\left(I_{X}-T\right) \leq p$;

(2.8.7) $T$ satisfies condition $\mathcal{E}(k, p)$ for some $k \in \mathbb{Z}_{+}, \delta\left(I_{X}-T\right)<\infty$ and $N\left(\left(I_{X}-T\right)^{p}\right)$ has an algebraic complement which is invariant under $T$;

(2.8.8) $\quad n^{-p}\left\|T^{n}\right\|_{L(X)} \rightarrow 0$ as $n \rightarrow \infty$ and $I_{X}-T$ is a Kato decomposable operator;

(2.8.9) $\quad n^{-p}\left\|T^{n}\right\|_{L(X)} \rightarrow 0$ as $n \rightarrow \infty$ and $\mathcal{R}\left(\left(I_{X}-T\right)^{k}\right)+\mathcal{N}\left(\left(I_{X}-T\right)^{j}\right)$ is closed for some $(k, j) \in \mathbb{N} \times \mathbb{N}$ satisfying $k \geq p$;

$n^{-p}\left\|T^{n}\right\|_{L(X)} \rightarrow 0$ as $n \rightarrow \infty$ and $\mathcal{R}\left(\left(I_{X}-T\right)^{k}\right)+\mathcal{N}\left(\left(I_{X}-T\right)^{j}\right)$ is closed for every $(k, j) \in \mathbb{N} \times \mathbb{N}$ satisfying $k+j \geq p$.

Moreover, if the equivalent conditions (2.8.1)-(2.8.10) are satisfied, then

$$
\left\|\frac{1}{n^{p}} \sum_{k=0}^{n-1} T^{k}-\frac{1}{p !}\left(T-I_{X}\right)^{p-1} P\right\|_{L(X)} \rightarrow 0 \quad \text { as } n \rightarrow \infty
$$

(where $P$ denotes the projection of $X$ onto $\mathcal{N}\left(\left(I_{X}-T\right)^{p}\right)$ along $\left.\mathcal{R}\left(\left(I_{X}-T\right)^{p}\right)\right)$.

Notice that, for $p=1$, the requirement of $(2.8 .1)$ that $\mathcal{R}\left(\left(I_{X}-T\right)^{p-1}\right)+$ $\mathcal{N}\left(I_{X}-T\right)$ be closed is automatically satisfied and so (2.8.1) reduces to convergence of $n^{-1} \sum_{k=0}^{n-1} T^{k}$ in $L(X)$, that is, to uniform ergodicity of $T$.

We remark that Theorem 2.8, besides generalizing the uniform ergodic theorem, generalizes also Theorem 6 of $[\mathrm{LM}]$, as condition $(\mathcal{E}-p)$ implies convergence of $n^{-p}\left\|T^{n}\right\|_{L(X)}$ to zero by Theorem 2.6 (see also [Bu1, comments following 3.4]).

We recall that if $T$ is a bounded linear operator on a Banach space $X$, then

$$
\mathcal{R}\left(T^{k-j}\right)+\mathcal{N}\left(T^{h+j}\right)=\left(T^{j}\right)^{-1}\left(\mathcal{R}\left(T^{k}\right)+\mathcal{N}\left(T^{h}\right)\right)
$$

for all $(k, h) \in \mathbb{N} \times \mathbb{N}$ and $j=0, \ldots, k$.

Hence ([Bu1, 3.1], [MM, Lemma 10])

$$
\text { if } \mathcal{R}\left(T^{n}\right)+\mathcal{N}\left(T^{m}\right) \text { is closed for some }(n, m) \in \mathbb{N} \times \mathbb{N} \text {, then } \mathcal{R}\left(T^{n-j}\right)
$$
$+\mathcal{N}\left(T^{m+j}\right)$ is closed for all $j=0, \ldots, n$.

Another result that relates closedness of different sums of ranges and kernels of iterates of a bounded linear operator is the following theorem by S. Grabiner and J. Zemánek, which we will use in the next section to obtain a new condition, equivalent to (2.8.1)-(2.8.10). 
Theorem 2.10 ([GZ, 2.1]). Let $X$ be a Banach space and $T \in L(X)$. If $\alpha(T) \in \mathbb{N}$ and $\mathcal{R}\left(T^{k}\right)+\mathcal{N}\left(T^{j}\right)$ is closed for some $(k, j) \in \mathbb{Z}_{+} \times \mathbb{N}$ satisfying $k+j>\alpha(T)$, then $\mathcal{R}\left(T^{n}\right)+\mathcal{N}\left(T^{m}\right)$ is closed for all $(n, m) \in \mathbb{N} \times \mathbb{N}$ satisfying $n+m \geq \alpha(T)$.

We remark that in the special case of a Hilbert space operator, Theorem 2.10 can also be derived from [Lab, Proposition 3.3.1] — which is a result in the Hilbert space setting - together with (2.9) and [TL, IV, 5.10 and V, 6.3].

3. Results and examples. We begin by remarking that the conditions of Theorem 2.8 - except (2.8.2) and (2.8.3) - can also be considered for an operator on a real Banach space. Indeed, these conditions are equivalent in the real case as well as in the complex one, as we are going to observe in Theorem 3.1 below.

For every real Banach space $X$, let $\widetilde{X}$ denote the complexification of $X$, that is, the complex vector space of all $x+i y, x, y \in X$, where the vector space operations are induced in the canonical way by the corresponding operations on $X$, endowed with the complete norm defined by

$$
\begin{aligned}
\|x+i y\|_{\widetilde{X}}=\sup \left\{\|(\cos \theta) x+(\sin \theta) y\|_{X}: \theta\right. & \in[0,2 \pi)\} \\
& \text { for each }(x, y) \in X \times X .
\end{aligned}
$$

Also, for every $T \in L(X)$, let $\widetilde{T}$ denote the complex extension of $T$, that is, the bounded linear operator on $\widetilde{X}$ defined by

$$
\widetilde{T}(x+i y)=T x+i T y \quad \text { for each }(x, y) \in X \times X
$$

(see $\left[\mathrm{S}\right.$, p. 261] for these definitions). It is easily seen that $\|\widetilde{T}\|_{L(\widetilde{X})}=$ $\|T\|_{L(X)}, \mathcal{N}(\widetilde{T})=\mathcal{N}(T)+i \mathcal{N}(T), \mathcal{R}(\widetilde{T})=\mathcal{R}(T)+i \mathcal{R}(T),(\widetilde{T})^{n}=\left(T^{n}\right)^{\sim}$ for every $n \in \mathbb{N}$ and $\widetilde{I}_{X}=I_{\widetilde{X}}$.

Theorem 3.1. Let $p \in \mathbb{Z}_{+}, X$ be a real Banach space and $T \in L(X)$. Then conditions (2.8.1) and (2.8.4)-(2.8.10) are equivalent for T. Furthermore, $T$ satisfies conditions (2.8.1) and (2.8.4)-(2.8.10) if and only if $\widetilde{T}$ satisfies (2.8.1)-(2.8.10). Finally, if T satisfies conditions (2.8.1) and (2.8.4)(2.8.10), then

$$
\left\|\frac{1}{n^{p}} \sum_{k=0}^{n-1} T^{k}-\frac{1}{p !}\left(T-I_{X}\right)^{p-1} P\right\|_{L(X)} \rightarrow 0 \quad \text { as } n \rightarrow \infty
$$

(where $P$ denotes the projection of $X$ onto $\mathcal{N}\left(\left(I_{X}-T\right)^{p}\right)$ along $\left.\mathcal{R}\left(\left(I_{X}-T\right)^{p}\right)\right)$.

Proof. It is not difficult to verify that each of conditions (2.8.1), (2.8.4), (2.8.5), (2.8.6), (2.8.9) and (2.8.10) is satisfied by $T$ if and only if it is satisfied by $\widetilde{T}$. From Theorem 2.8 it follows that, for any $h, k \in\{1,4,5,6,9,10\}$, 
$T$ satisfies (2.8.h) if and only if it satisfies (2.8.k). Furthermore, if $T$ satisfies the equivalent conditions (2.8.1), (2.8.4)-(2.8.6) and (2.8.9)-(2.8.10), it clearly also fulfils (2.8.7) and (2.8.8). Conversely, if $T$ satisfies one of conditions (2.8.7)-(2.8.8), it is easily seen that also $\widetilde{T}$ does. Hence, by Theorem $2.8, \widetilde{T}$ satisfies the equivalent conditions (2.8.1), (2.8.4)-(2.8.6) and (2.8.9)-(2.8.10). Then, as remarked above, also $T$ does.

We have thus proved that each of conditions (2.8.1) and (2.8.4)-(2.8.10) is satisfied by $T$ if and only if also the remaining ones are, and that $T$ satisfies the equivalent conditions (2.8.1) and (2.8.4)-(2.8.10) if and only if $\widetilde{T}$ satisfies the equivalent conditions $(2.8 .1)-(2.8 .10)$.

Now suppose that the equivalent conditions (2.8.1) and (2.8.4)-(2.8.10) are satisfied by $T$. Then the equivalent conditions (2.8.1)-(2.8.10) are satisfied by $\widetilde{T}$. In particular, we have $X=\mathcal{N}\left(\left(I_{X}-T\right)^{p}\right) \oplus \mathcal{R}\left(\left(I_{X}-T\right)^{p}\right)$ and $\widetilde{X}=\mathcal{N}\left(\left(I_{\widetilde{X}}-\widetilde{T}\right)^{p}\right) \oplus \mathcal{R}\left(\left(I_{\widetilde{X}}-\widetilde{T}\right)^{p}\right)$. Notice also that the projection of $\widetilde{X}$ onto $\mathcal{N}\left(\left(I_{\widetilde{X}}-\widetilde{T}\right)^{p}\right)$ along $\mathcal{R}\left(\left(I_{\widetilde{X}}-\widetilde{T}\right)^{p}\right)$ coincides with $\widetilde{P}$. Hence the desired result follows from Theorem 2.8 .

Definition 3.2. Let $p \in \mathbb{Z}_{+}$. We call a bounded linear operator $T$ on a Banach space $X$ uniformly $p$-ergodic if $n^{-p} \sum_{k=0}^{n-1} T^{k}$ converges in $L(X)$ and $\mathcal{R}\left(\left(I_{X}-T\right)^{p-1}\right)+\mathcal{N}\left(I_{X}-T\right)$ is closed.

Notice that $T$ is uniformly 1-ergodic if and only if it is uniformly ergodic.

Clearly, if the Banach space $X$ is complex (respectively, real), then $T$ is uniformly $p$-ergodic if and only if it satisfies the equivalent conditions (2.8.1)-(2.8.10) (respectively, (2.8.1) and (2.8.4)-(2.8.10)). Moreover, from Theorem 3.1 it follows that a bounded linear operator $T$ on a real Banach space is uniformly $p$-ergodic if and only if $\widetilde{T}$ is uniformly $p$-ergodic.

Proposition 3.3. Let $X$ be a Banach space and $T \in L(X)$. If $T$ is uniformly $p_{0}$-ergodic for some $p_{0} \in \mathbb{Z}_{+}$, then $T$ is uniformly $p$-ergodic for all $p \in \mathbb{Z}_{+}$satisfying $p \geq p_{0}$.

Proof. It suffices for instance to remark that since condition (2.8.5) is satisfied for $p=p_{0}$, it is also satisfied for all positive integers $p$ satisfying $p \geq p_{0}$.

Now we are going to derive a further characterization of uniform $p$ ergodicity from Theorem 2.10 .

Theorem 3.4. Let $p \in \mathbb{Z}_{+}, X$ be a Banach space and $T \in L(X)$. Then $T$ is uniformly p-ergodic if and only if it satisfies the following condition:

$$
\begin{aligned}
& n^{-p}\left\|T^{n}\right\|_{L(X)} \rightarrow 0 \text { as } n \rightarrow \infty \text { and } \mathcal{R}\left(\left(I_{X}-T\right)^{k}\right)+\mathcal{N}\left(\left(I_{X}-T\right)^{j}\right) \\
& \text { is closed for some }(k, j) \in \mathbb{Z}_{+} \times \mathbb{N} \text { satisfying } k+j>p .
\end{aligned}
$$


Proof. If $T$ is uniformly $p$-ergodic, it satisfies condition (2.8.10), and consequently also (3.4.1).

Conversely, assume that (3.4.1) is satisfied. Since $n^{-p}\left\|T^{n}\right\|_{L(X)} \rightarrow 0$ as $n \rightarrow \infty$, from (2.7) it follows that $\alpha\left(I_{X}-T\right) \leq p$. By applying Theorem 2.10, we conclude that $\mathcal{R}\left(\left(I_{X}-T\right)^{k}\right)+\mathcal{N}\left(\left(I_{X}-T\right)^{j}\right)$ is closed for all $(k, j) \in \mathbb{N} \times \mathbb{N}$ satisfying $k+j \geq p$. Hence $T$ satisfies (2.8.10) and the proof is finished.

We point out that, since norm convergence of $n^{-p} T^{n}$ to zero cannot be replaced by strong convergence to zero in condition (2.8.10), as the example constructed in [Bu1, 3.8] shows (indeed, as remarked in [Bu1], [Bu1, 3.8] also shows that the same holds for each of conditions (2.8.2), (2.8.5), (2.8.8) and (2.8.9), and that condition $\mathcal{E}(k, p)$ can be replaced by $\mathcal{S}(1, p)$ in none of conditions $(2.8 .3),(2.8 .4),(2.8 .6)$ and $(2.8 .7))$, it follows that norm convergence of $n^{-p} T^{n}$ to zero cannot be replaced by strong convergence to zero in condition (3.4.1), either.

Now let $X$ be a complex Banach space, $T \in L(X)$ and $p \in \mathbb{Z}_{+}$. It is well known that if $\sigma(T)=\{1\}$, we have $\left(I_{X}-T\right)^{p}=0_{L(X)}$ if and only if $1 \in$ $\mathcal{P}_{p}(T)$ (see Theorem 2.2 and $[\mathrm{TL}, \mathrm{V}, 10.6]$ ). Also, it follows from a theorem by E. Hille (see for instance [HP, Theorem 4.10.1]) that $\left(I_{X}-T\right)^{p}=0_{L(X)}$ implies $n^{-p}\left\|T^{n}\right\|_{L(X)} \rightarrow 0$ as $n \rightarrow \infty$ (indeed, $\left(I_{X}-T\right)^{p}=0_{L(X)}$ implies

$$
\frac{1}{n^{p-1}} T^{n} \rightarrow \frac{1}{(p-1) !}\left(T-I_{X}\right)^{p-1}
$$

in $L(X)$ as $n \rightarrow \infty$; see [W, Hilfssatz 3]). Hence from Theorems 2.8 and 3.4 we obtain the following result.

Corollary 3.5. Let $p \in \mathbb{Z}_{+}, X$ be a complex Banach space and $T \in$ $L(X)$. Then $\left(I_{X}-T\right)^{p}=0_{L(X)}$ if and only if $\sigma(T)=\{1\}, n^{-p}\left\|T^{n}\right\|_{L(X)} \rightarrow 0$ as $n \rightarrow \infty$ and $\mathcal{R}\left(\left(I_{X}-T\right)^{k}\right)+\mathcal{N}\left(\left(I_{X}-T\right)^{j}\right)$ is closed for some $(k, j) \in$ $\mathbb{Z}_{+} \times \mathbb{N}$ satisfying either $k=p$ and $j=0$, or $k+j>p$.

We remark that Corollary 3.5 generalizes Corollary 2 of [MZ], which states that $T=I_{X}$ if and only if $\sigma(T)=\{1\}, n^{-1}\left\|T^{n}\right\|_{L(X)} \rightarrow 0$ as $n \rightarrow \infty$ and $\mathcal{R}\left(\left(I_{X}-T\right)^{k}\right)$ is closed for some $k \in \mathbb{Z}_{+}$. Moreover, since convergence of $n^{-p}\left\|T^{n}\right\|_{L(X)}$ to zero is less restrictive than condition $(\mathcal{E}-p)$ (see Theorem 2.6 and comments thereafter), it follows that Corollary 3.5 is also an improvement of Corollary 7 of $[\mathrm{LM}]$, in which $\left(I_{X}-T\right)^{p}$ is proved to be zero if and only if $\sigma(T)=\{1\}, T$ satisfies condition $(\mathcal{E}-p)$ and $\mathcal{R}\left(\left(I_{X}-T\right)^{k}\right)$ is closed for some $k \geq p$.

We observe that for $k+j>p$, convergence of $n^{-p}\left\|T^{n}\right\|_{L(X)}$ to zero can be replaced in Corollary 3.5 by the weaker condition $\alpha\left(I_{X}-T\right) \leq p$, although in Theorem 3.4 it cannot even be replaced by strong convergence of $n^{-p} T^{n}$ to zero (which is a stronger condition than $\alpha\left(I_{X}-T\right) \leq p$, by 
(2.7)). Indeed, if $\alpha\left(I_{X}-T\right) \leq p$ and $\mathcal{R}\left(\left(I_{X}-T\right)^{k}\right)+\mathcal{N}\left(\left(I_{X}-T\right)^{j}\right)$ is closed for some $(k, j) \in \mathbb{Z}_{+} \times \mathbb{N}$ satisfying $k+j>p$, from Theorem 2.10 we conclude that $\mathcal{R}\left(\left(I_{X}-T\right)^{p+1}\right)$ is closed. Since 1 is a boundary point of $\sigma(T)$ (as $\sigma(T)=\{1\}$ ), from [Lay, 2.7] we obtain $1 \in \mathcal{P}_{p}(T)$ (see also [GZ, 2.3]). This, together with $\sigma(T)=\{1\}$, yields $\left(I_{X}-T\right)^{p}=0_{L(X)}$, as recalled in the comments preceding Corollary 3.5. On the contrary, for $k=p$ and $j=0$, the requirement in Corollary 3.5 that $n^{-p}\left\|T^{n}\right\|_{L(X)} \rightarrow 0$ as $n \rightarrow \infty$ cannot be replaced by $\alpha\left(I_{X}-T\right) \leq p$ : indeed, in [Bu1, 3.5] we constructed a bounded linear operator $T$ on $\ell_{2} \times \ell_{2}$ such that $\sigma(T)=\{1\}, \alpha\left(I_{\ell_{2} \times \ell_{2}}-T\right)=1$, $\mathcal{R}\left(I_{\ell_{2} \times \ell_{2}}-T\right)$ is closed and nevertheless $1 \notin \mathcal{P}(T)$. Actually, for $k=p$ and $j=0$, norm convergence of $n^{-p} T^{n}$ to zero cannot even be replaced by strong convergence to zero, as proved by the example with spectrum $\{1\}$ constructed in [GZ, 1.3].

The following example shows that when $p \geq 2$ and $1 \leq k<p$, the condition " $k+j>p$ " cannot be replaced by " $k+j \geq p$ " in (3.4.1). Indeed, we will construct a bounded linear operator $T$ on a Hilbert space $X$ such that $n^{-2}\left\|T^{n}\right\|_{L(X)}$ converges to zero as $n \rightarrow \infty, \mathcal{R}\left(I_{X}-T\right)+\mathcal{N}\left(I_{X}-T\right)$ is closed and nevertheless $T$ is not uniformly 2-ergodic (actually, it is uniformly $p$-ergodic for no $p \in \mathbb{Z}_{+}$).

We will denote the scalar product in any Hilbert space by $\langle\cdot, \cdot\rangle$. Also, for every subset $S$ of a linear space $V$, let $\operatorname{Span}(V)$ denote the linear subspace of $V$ spanned by $S$.

EXAmPle 3.6. Let $X$ be an infinite-dimensional separable Hilbert space, $\left\{e_{n}\right\}_{n \in \mathbb{N}}$ be an orthonormal basis of $X$ and $\left(\theta_{n}\right)_{n \in \mathbb{N}}$ be a sequence in $(0, \pi / 2]$ such that $\theta_{n} \rightarrow 0$ as $n \rightarrow \infty$. For every $n \in \mathbb{N}$, let $f_{n} \in X$ be defined by

$$
f_{n}=-\left(\cos \theta_{n}\right) e_{3 n+1}+\left(\sin \theta_{n}\right) e_{3 n+2} .
$$

Notice that $\left\|f_{n}\right\|_{X}=1$. We also remark that the set $\left\{e_{3 n}, e_{3 n+1}, f_{n}\right\}$ is linearly independent for every $n \in \mathbb{N}$ : indeed, if $\lambda_{0}, \lambda_{1}, \lambda_{2} \in \mathbb{K}$ satisfy

$$
\begin{aligned}
0_{X} & =\lambda_{0} e_{3 n}+\lambda_{1} e_{3 n+1}+\lambda_{2} f_{n} \\
& =\lambda_{0} e_{3 n}+\left(\lambda_{1}-\lambda_{2} \cos \theta_{n}\right) e_{3 n+1}+\lambda_{2}\left(\sin \theta_{n}\right) e_{3 n+2},
\end{aligned}
$$

then since the set $\left\{e_{3 n}, e_{3 n+1}, e_{3 n+2}\right\}$ is linearly independent it follows that

$$
\left\{\begin{array}{l}
\lambda_{0}=0, \\
\lambda_{1}=\lambda_{2} \cos \theta_{n}, \\
\lambda_{2} \sin \theta_{n}=0,
\end{array}\right.
$$

which, since $\theta_{n} \in(0, \pi / 2]$ and consequently $\sin \theta_{n}>0$, gives $\lambda_{0}=\lambda_{1}=$ $\lambda_{2}=0$.

For each $n \in \mathbb{N}$, we set $X_{n}=\operatorname{Span}\left(\left\{e_{3 n}, e_{3 n+1}, f_{n}\right\}\right)$. Since $X_{n}$ is threedimensional and is contained in $\operatorname{Span}\left(\left\{e_{3 n}, e_{3 n+1}, e_{3 n+2}\right\}\right)$, it follows that 
$X_{n}=\operatorname{Span}\left(\left\{e_{3 n}, e_{3 n+1}, e_{3 n+2}\right\}\right)$. For every $n \in \mathbb{N}$, let $T_{n} \in L\left(X_{n}\right)$ be defined by

$$
\begin{aligned}
T_{n} e_{3 n} & =e_{3 n}, \quad T_{n} e_{3 n+1}=-\sqrt{1-\cos \theta_{n}} e_{3 n}+e_{3 n+1}, \\
T_{n} f_{n} & =\left(1-\sqrt{1-\cos \theta_{n}}\right) f_{n} .
\end{aligned}
$$

We are going to provide an upper bound for $\left\|T_{n}\right\|_{L\left(X_{n}\right)}$.

For every $(x, y, z) \in \mathbb{K}^{3}$, we have

$$
\begin{gathered}
\left\|x e_{3 n}+y e_{3 n+1}+z f_{n}\right\|_{X}^{2} \\
=\left\|x e_{3 n}+\left(y-z \cos \theta_{n}\right) e_{3 n+1}+z\left(\sin \theta_{n}\right) e_{3 n+2}\right\|_{X}^{2} \\
=|x|^{2}+\left|y-z \cos \theta_{n}\right|^{2}+|z|^{2} \sin ^{2} \theta_{n},
\end{gathered}
$$

from which we obtain

$$
\begin{aligned}
\left\|x e_{3 n}+y e_{3 n+1}+z f_{n}\right\|_{X}^{2} & \\
& \geq|x|^{2}+\left(|y|-|z| \cos \theta_{n}\right)^{2}+|z|^{2} \sin ^{2} \theta_{n} \\
& =|x|^{2}+|y|^{2}+|z|^{2}-2|y||z| \cos \theta_{n} \\
& \geq|x|^{2}+\left(1-\cos \theta_{n}\right)\left(|y|^{2}+|z|^{2}\right)
\end{aligned}
$$

(as $2|y||z| \leq|y|^{2}+|z|^{2}$ ). We also remark that

$$
\begin{aligned}
& T_{n}\left(x e_{3 n}+y e_{3 n+1}+z f_{n}\right) \\
& =x e_{3 n}+y\left(e_{3 n+1}-\sqrt{1-\cos \theta_{n}} e_{3 n}\right)+z\left(1-\sqrt{1-\cos \theta_{n}}\right) f_{n} \\
& =x e_{3 n}+y e_{3 n+1}+z f_{n}-\sqrt{1-\cos \theta_{n}}\left(y e_{3 n}+z f_{n}\right) \\
& =\left(x-y \sqrt{1-\cos \theta_{n}}\right) e_{3 n}+y e_{3 n+1}+z\left(1-\sqrt{1-\cos \theta_{n}}\right) f_{n} .
\end{aligned}
$$

Since the set $\left\{e_{3 n}, f_{n}\right\}$ is orthonormal, (3.6.2) and (3.6.3) yield

$$
\begin{aligned}
\| T_{n}\left(x e_{3 n}+\right. & \left.y e_{3 n+1}+z f_{n}\right) \|_{X} \\
& =\left\|x e_{3 n}+y e_{3 n+1}+z f_{n}-\sqrt{1-\cos \theta_{n}}\left(y e_{3 n}+z f_{n}\right)\right\|_{X} \\
& \leq\left\|x e_{3 n}+y e_{3 n+1}+z f_{n}\right\|_{X}+\sqrt{1-\cos \theta_{n}}\left\|y e_{3 n}+z f_{n}\right\|_{X} \\
& =\left\|x e_{3 n}+y e_{3 n+1}+z f_{n}\right\|_{X}+\sqrt{\left(1-\cos \theta_{n}\right)\left(|y|^{2}+|z|^{2}\right)} \\
& \leq 2\left\|x e_{3 n}+y e_{3 n+1}+z f_{n}\right\|_{X} .
\end{aligned}
$$

We conclude that $\left\|T_{n}\right\|_{L\left(X_{n}\right)} \leq 2$.

For each $n \in \mathbb{N}$, let $P_{n}$ denote the orthogonal projection of $X$ onto $X_{n}$. Since $\left(X_{n}\right)_{n \in \mathbb{N}}$ is a sequence of pairwise orthogonal closed subspaces of $X$ and

$$
\operatorname{Span}\left(\bigcup_{n \in \mathbb{N}} X_{n}\right)=\operatorname{Span}\left(\left\{e_{n}\right\}_{n \in \mathbb{N}}\right),
$$


which is dense in $X$, we conclude (by applying, for instance, [Bu2, 5.3]) that for every $x \in X$ we have

$$
x=\sum_{n=0}^{\infty} P_{n} x, \quad\|x\|_{X}^{2}=\sum_{n=0}^{\infty}\left\|P_{n} x\right\|_{X}^{2},
$$

which gives

$$
\sum_{n=0}^{\infty}\left\|T_{n} P_{n} x\right\|_{X}^{2} \leq \sum_{n=0}^{\infty}\left\|T_{n}\right\|_{L(X)}^{2}\left\|P_{n} x\right\|_{X}^{2} \leq 4 \sum_{n=0}^{\infty}\left\|P_{n} x\right\|_{X}^{2}=4\|x\|_{X}^{2} .
$$

Since $\left(T_{n} P_{n} x\right)_{n \in \mathbb{N}}$ is an orthogonal sequence in $X$, we conclude that the series $\sum_{n=0}^{\infty} T_{n} P_{n} x$ converges in $X$ and $\left\|\sum_{n=0}^{\infty} T_{n} P_{n} x\right\|_{X} \leq 2\|x\|_{X}$.

Let us consider the bounded linear operator

$$
T: X \ni x \mapsto \sum_{n=0}^{\infty} T_{n} P_{n} x \in X .
$$

Notice that $\|T\|_{L(X)} \leq 2$. Moreover,

$$
T x=T_{n} x \quad \text { for every } x \in X_{n} \text { and for every } n \in \mathbb{N} .
$$

We begin by proving that $T$ is uniformly $p$-ergodic for no $p \in \mathbb{Z}_{+}$. Suppose first $\mathbb{K}=\mathbb{C}$. We remark that, for each $n \in \mathbb{N}$,

$$
T e_{3 n}=T_{n} e_{3 n}=e_{3 n}, \quad T f_{n}=T_{n} f_{n}=\left(1-\sqrt{1-\cos \theta_{n}}\right) f_{n} .
$$

Hence $1 \in \sigma(T)$ and $1-\sqrt{1-\cos \theta_{n}} \in \sigma(T)$ for every $n \in \mathbb{N}$. Since $\theta_{n} \in$ $(0, \pi / 2]$, it follows that $\cos \theta_{n} \in[0,1)$ and consequently $1-\sqrt{1-\cos \theta_{n}} \in$ $[0,1)$. Furthermore, since $\theta_{n} \rightarrow 0$ as $n \rightarrow \infty$, it follows that $1-\sqrt{1-\cos \theta_{n}}$ $\rightarrow 1$ as $n \rightarrow \infty$. We conclude that 1 is not an isolated point of $\sigma(T)$, and consequently is not a pole of the resolvent of $T$. Hence $1 \notin \mathcal{P}(T)$. For $\mathbb{K}=\mathbb{R}$, the same argument yields $1 \notin \mathcal{P}(\widetilde{T})$. Now from Theorem 2.8 for $\mathbb{K}=\mathbb{C}$, and from Theorem 3.1 for $\mathbb{K}=\mathbb{R}$, it follows that $T$ is uniformly $p$-ergodic for no $p \in \mathbb{Z}_{+}$. In particular, $T$ is not uniformly 2 -ergodic.

Now we prove that $\mathcal{R}\left(I_{X}-T\right)+\mathcal{N}\left(I_{X}-T\right)$ is closed. Since it contains $\mathcal{N}\left(I_{X}-T\right)$, which is closed, it suffices to prove that $\left(\mathcal{R}\left(I_{X}-T\right)+\mathcal{N}\left(I_{X}-T\right)\right) \cap$ $\left(\mathcal{N}\left(I_{X}-T\right)\right)^{\perp}$ is closed. Let $P$ denote the orthogonal projection of $X$ onto $\left(\mathcal{N}\left(I_{X}-T\right)\right)^{\perp}$. We remark that since $\mathcal{R}\left(I_{X}-T\right)+\mathcal{N}\left(I_{X}-T\right) \supset \mathcal{N}\left(I_{X}-T\right)$, we have

$$
\begin{aligned}
\left(\mathcal{R}\left(I_{X}-T\right)\right. & \left.+\mathcal{N}\left(I_{X}-T\right)\right) \cap\left(\mathcal{N}\left(I_{X}-T\right)\right)^{\perp} \\
& =P\left(\mathcal{R}\left(I_{X}-T\right)+\mathcal{N}\left(I_{X}-T\right)\right)=\mathcal{R}\left(P\left(I_{X}-T\right)\right) .
\end{aligned}
$$

Notice also that

$$
\mathcal{N}\left(P\left(I_{X}-T\right)\right)=\left\{x \in X:\left(I_{X}-T\right) x \in \mathcal{N}(P)\right\}=\mathcal{N}\left(\left(I_{X}-T\right)^{2}\right)
$$


Since the subspaces $X_{n}, n \in \mathbb{N}$, are pairwise orthogonal, from (3.6.4) it follows that for each $x \in X$ we have

$$
\begin{aligned}
x \in \mathcal{N}\left(I_{X}-T\right) & \Leftrightarrow \sum_{n=0}^{\infty} T_{n} P_{n} x=\sum_{n=0}^{\infty} P_{n} x \\
& \Leftrightarrow T_{n} P_{n} x=P_{n} x \text { for every } n \in \mathbb{N} \\
& \Leftrightarrow P_{n} x \in \mathcal{N}\left(I_{X_{n}}-T_{n}\right) \text { for every } n \in \mathbb{N} .
\end{aligned}
$$

Consequently, for each $x \in X$,

$$
\begin{aligned}
& x \in \mathcal{N}\left(\left(I_{X}-T\right)^{2}\right) \Leftrightarrow\left(I_{X}-T\right) x \in \mathcal{N}\left(I_{X}-T\right) \\
& \quad \Leftrightarrow \mathcal{N}\left(I_{X_{n}}-T_{n}\right) \ni P_{n}\left(I_{X}-T\right) x=\left(I_{X_{n}}-T_{n}\right) P_{n} x \text { for all } n \in \mathbb{N} \\
& \quad \Leftrightarrow P_{n} x \in \mathcal{N}\left(\left(I_{X_{n}}-T_{n}\right)^{2}\right) \text { for all } n \in \mathbb{N} .
\end{aligned}
$$

We prove that

$$
\left(\mathcal{N}\left(I_{X}-T\right)\right)^{\perp}=\left\{x \in X: P_{n} x \in\left(\mathcal{N}\left(I_{X_{n}}-T_{n}\right)\right)^{\perp} \text { for all } n \in \mathbb{N}\right\} .
$$

We first observe that, by (3.6.7), we have $\mathcal{N}\left(I_{X_{n}}-T_{n}\right) \subset \mathcal{N}\left(I_{X}-T\right)$ for every $n \in \mathbb{N}$. Consequently, $\left(\mathcal{N}\left(I_{X}-T\right)\right)^{\perp} \subset\left(\mathcal{N}\left(I_{X_{n}}-T_{n}\right)\right)^{\perp}$ for each $n \in \mathbb{N}$, which gives $0=\langle x, y\rangle=\sum_{k=0}^{\infty}\left\langle P_{k} x, y\right\rangle=\left\langle P_{n} x, y\right\rangle$ for all $x \in\left(\mathcal{N}\left(I_{X}-T\right)\right)^{\perp}$ and $y \in \mathcal{N}\left(I_{X_{n}}-T_{n}\right)$. Hence

$$
\left(\mathcal{N}\left(I_{X}-T\right)\right)^{\perp} \subset\left\{x \in X: P_{n} x \in\left(\mathcal{N}\left(I_{X_{n}}-T_{n}\right)\right)^{\perp} \text { for all } n \in \mathbb{N}\right\} .
$$

On the other hand, for each $x \in X$ such that $P_{n} x \in\left(\mathcal{N}\left(I_{X_{n}}-T_{n}\right)\right)^{\perp}$ for all $n \in \mathbb{N}$, we have

$$
\langle x, y\rangle=\left\langle\sum_{n=0}^{\infty} P_{n} x, \sum_{n=0}^{\infty} P_{n} y\right\rangle=\sum_{n=0}^{\infty}\left\langle P_{n} x, P_{n} y\right\rangle=0
$$

$$
\text { for every } y \in \mathcal{N}\left(I_{X}-T\right)
$$

(as $P_{n} y \in \mathcal{N}\left(I_{X_{n}}-T_{n}\right)$ for all $n \in \mathbb{N}$ by (3.6.7)). This proves the opposite inclusion and gives (3.6.9).

For each $n \in \mathbb{N}$, let $Q_{n}$ denote the orthogonal projection of $X$ onto $X_{n} \cap\left(\mathcal{N}\left(I_{X_{n}}-T_{n}\right)\right)^{\perp}$. Since $\mathcal{R}\left(Q_{n}\right) \subset X_{n}=\mathcal{R}\left(P_{n}\right)$ for every $n \in \mathbb{N}$, from (3.6.4) it follows that

$$
\sum_{n=0}^{\infty}\left\|Q_{n} x\right\|_{X}^{2} \leq \sum_{n=0}^{\infty}\left\|P_{n} x\right\|_{X}^{2}=\|x\|_{X}^{2} \quad \text { for every } x \in X .
$$

Since the ranges of the projections $Q_{n}, n \in \mathbb{N}$, are pairwise orthogonal, we conclude that the series $\sum_{n=0}^{\infty} Q_{n} x$ converges in $X$ for every $x \in X$. We prove that

$$
P x=\sum_{n=0}^{\infty} Q_{n} x \quad \text { for every } x \in X .
$$


Let $x \in X$. We remark that for each $n \in \mathbb{N}$ we have

$$
P_{n} \sum_{k=0}^{\infty} Q_{k} x=\sum_{k=0}^{\infty} P_{n} Q_{k} x=Q_{n} x \in\left(\mathcal{N}\left(I_{X_{n}}-T_{n}\right)\right)^{\perp}
$$

and

$$
\begin{aligned}
P_{n}\left(x-\sum_{k=0}^{\infty} Q_{k} x\right) & =P_{n} x-Q_{n} x \\
& =\left(I_{X}-Q_{n}\right) P_{n} x \in X_{n} \cap\left(X_{n} \cap\left(\mathcal{N}\left(I_{X_{n}}-T_{n}\right)\right)^{\perp}\right)^{\perp} \\
& =X_{n} \cap\left(X_{n}^{\perp} \oplus \mathcal{N}\left(I_{X_{n}}-T_{n}\right)\right)=\mathcal{N}\left(I_{X_{n}}-T_{n}\right) .
\end{aligned}
$$

So $\sum_{n=0}^{\infty} Q_{n} x \in\left(\mathcal{N}\left(I_{X}-T\right)\right)^{\perp}$ by (3.6.9) and $x-\sum_{n=0}^{\infty} Q_{n} x \in \mathcal{N}\left(I_{X}-T\right)$ by (3.6.7). We conclude that $\sum_{n=0}^{\infty} Q_{n} x=P x$, which establishes (3.6.10).

Since $\left(I_{X_{n}}-T_{n}\right) e_{3 n}=0_{X}$ and $\left(I_{X_{n}}-T_{n}\right) e_{3 n+1}=\sqrt{1-\cos \theta_{n}} e_{3 n} \in$ $\mathcal{N}\left(I_{X_{n}}-T_{n}\right) \backslash\left\{0_{X}\right\}$ and $f_{n} \in \mathcal{N}\left(\left(1-\sqrt{1-\cos \theta_{n}}\right) I_{X_{n}}-T_{n}\right)$, which has zero intersection with $\mathcal{N}\left(\left(I_{X_{n}}-T_{n}\right)^{2}\right)$ as $1-\sqrt{1-\cos \theta_{n}} \neq 1$, it follows that

$$
\begin{aligned}
\mathcal{N}\left(I_{X_{n}}-T_{n}\right) & =\operatorname{Span}\left(\left\{e_{3 n}\right\}\right), \\
\mathcal{N}\left(\left(I_{X_{n}}-T_{n}\right)^{2}\right) & =\operatorname{Span}\left(\left\{e_{3 n}, e_{3 n+1}\right\}\right) \quad \text { for all } n \in \mathbb{N} .
\end{aligned}
$$

Notice also that

$$
\begin{aligned}
(3.6 .12) X_{n} \cap\left(\mathcal{N}\left(I_{X_{n}}-T_{n}\right)\right)^{\perp} & =\operatorname{Span}\left(\left\{e_{3 n+1}, e_{3 n+2}\right\}\right) \\
& =\operatorname{Span}\left(\left\{e_{3 n+1}, f_{n}\right\}\right) \quad \text { for all } n \in \mathbb{N} .
\end{aligned}
$$

Let $x \in X$. Then there exists a unique triple $\left(\left(x_{n}\right)_{n \in \mathbb{N}},\left(y_{n}\right)_{n \in \mathbb{N}},\left(z_{n}\right)_{n \in \mathbb{N}}\right)$ of scalar sequences such that $P_{n} x=x_{n} e_{3 n}+y_{n} e_{3 n+1}+z_{n} f_{n}$ for every $n \in \mathbb{N}$. Notice that, by (3.6.1) and (3.6.4), we have

$$
\begin{aligned}
\|x\|_{X}^{2} & =\sum_{n=0}^{\infty}\left\|P_{n} x\right\|_{X}^{2}=\sum_{n=0}^{\infty}\left\|x_{n} e_{3 n}+y_{n} e_{3 n+1}+z_{n} f_{n}\right\|_{X}^{2} \\
& =\sum_{n=0}^{\infty}\left(\left|x_{n}\right|^{2}+\left|y_{n}-z_{n} \cos \theta_{n}\right|^{2}+\left|z_{n}\right|^{2} \sin ^{2} \theta_{n}\right) .
\end{aligned}
$$

Hence each of the scalar sequences $\left(x_{n}\right)_{n \in \mathbb{N}},\left(y_{n}-z_{n} \cos \theta_{n}\right)_{n \in \mathbb{N}}$ and $\left(z_{n} \sin \theta_{n}\right)_{n \in \mathbb{N}}$ is square summable.

We remark that

$$
\begin{aligned}
P\left(I_{X}-T\right) x & =\sum_{n=0}^{\infty} Q_{n}\left(I_{X}-T\right) x=\sum_{n=0}^{\infty} Q_{n} \sum_{k=0}^{\infty}\left(I_{X_{k}}-T_{k}\right) P_{k} x \\
& =\sum_{n=0}^{\infty} Q_{n}\left(I_{X_{n}}-T_{n}\right) P_{n} x
\end{aligned}
$$




$$
\begin{aligned}
& =\sum_{n=0}^{\infty} Q_{n}\left(I_{X_{n}}-T_{n}\right)\left(x_{n} e_{3 n}+y_{n} e_{3 n+1}+z_{n} f_{n}\right) \\
& =\sum_{n=0}^{\infty} \sqrt{1-\cos \theta_{n}} Q_{n}\left(y_{n} e_{3 n}+z_{n} f_{n}\right)=\sum_{n=0}^{\infty} z_{n} \sqrt{1-\cos \theta_{n}} f_{n}
\end{aligned}
$$

by (3.6.4) and (3.6.10)-(3.6.12). Since $\left(f_{n}\right)_{n \in \mathbb{N}}$ is an orthonormal sequence, it follows that

$$
\left\|P\left(I_{X}-T\right) x\right\|_{X}^{2}=\sum_{n=0}^{\infty}\left|z_{n}\right|^{2}\left(1-\cos \theta_{n}\right) .
$$

Since $\left(x_{n} e_{3 n}+\left(y_{n}-z_{n} \cos \theta_{n}\right) e_{3 n+1}\right)_{n \in \mathbb{N}}$ is an orthogonal sequence in $X$ and

$$
\begin{aligned}
\sum_{n=0}^{\infty} \| x_{n} e_{3 n}+\left(y_{n}-z_{n} \cos \theta_{n}\right) & e_{3 n+1} \|_{X}^{2} \\
& =\sum_{n=0}^{\infty}\left(\left|x_{n}\right|^{2}+\left|y_{n}-z_{n} \cos \theta_{n}\right|^{2}\right) \leq\|x\|_{X}^{2}
\end{aligned}
$$

by (3.6.13), it follows that the series $\sum_{n=0}^{\infty}\left(x_{n} e_{3 n}+\left(y_{n}-z_{n} \cos \theta_{n}\right) e_{3 n+1}\right)$ converges in $X$. Furthermore, for each $n \in \mathbb{N}$, we have

$$
\begin{aligned}
P_{n} \sum_{k=0}^{\infty}\left(x_{k} e_{3 k}+\left(y_{k}-z_{k} \cos \theta_{k}\right) e_{3 k+1}\right) \\
=x_{n} e_{3 n}+\left(y_{n}-z_{n} \cos \theta_{n}\right) e_{3 n+1} \in \mathcal{N}\left(\left(I_{X_{n}}-T_{n}\right)^{2}\right)
\end{aligned}
$$

by (3.6.11). Consequently,

$$
\sum_{n=0}^{\infty}\left(x_{n} e_{3 n}+\left(y_{n}-z_{n} \cos \theta_{n}\right) e_{3 n+1}\right) \in \mathcal{N}\left(\left(I_{X}-T\right)^{2}\right)
$$

by (3.6.8). Hence

$$
\begin{aligned}
\operatorname{dist}\left(x, \mathcal{N}\left(P\left(I_{X}-T\right)\right)\right) \\
=\operatorname{dist}\left(x, \mathcal{N}\left(\left(I_{X}-T\right)^{2}\right)\right) \leq\left\|x-\sum_{n=0}^{\infty}\left(x_{n} e_{3 n}+\left(y_{n}-z_{n} \cos \theta_{n}\right) e_{3 n+1}\right)\right\|_{X} \\
=\left\|\sum_{n=0}^{\infty}\left(P_{n} x-\left(x_{n} e_{3 n}+\left(y_{n}-z_{n} \cos \theta_{n}\right) e_{3 n+1}\right)\right)\right\|_{X} \\
=\left\|\sum_{n=0}^{\infty}\left(x_{n} e_{3 n}+y_{n} e_{3 n+1}+z_{n} f_{n}-\left(x_{n} e_{3 n}+\left(y_{n}-z_{n} \cos \theta_{n}\right) e_{3 n+1}\right)\right)\right\|_{X} \\
=\left\|\sum_{n=0}^{\infty} z_{n}\left(f_{n}+\left(\cos \theta_{n}\right) e_{3 n+1}\right)\right\|_{X}=\left\|\sum_{n=0}^{\infty} z_{n}\left(\sin \theta_{n}\right) e_{3 n+2}\right\|_{X}
\end{aligned}
$$




$$
\begin{aligned}
& =\sqrt{\sum_{n=0}^{\infty}\left|z_{n}\right|^{2} \sin ^{2} \theta_{n}}=\sqrt{\sum_{n=0}^{\infty}\left|z_{n}\right|^{2}\left(1-\cos \theta_{n}\right)\left(1+\cos \theta_{n}\right)} \\
& \leq \sqrt{2} \sqrt{\sum_{n=0}^{\infty}\left|z_{n}\right|^{2}\left(1-\cos \theta_{n}\right)}=\sqrt{2}\left\|P\left(I_{X}-T\right) x\right\|_{X}
\end{aligned}
$$

by (3.6.4), (3.6.6) and (3.6.14). Now [TL, IV, 5.9] implies that $\mathcal{R}\left(P\left(I_{X}-T\right)\right)$ is closed, and so is $\left(\mathcal{R}\left(I_{X}-T\right)+\mathcal{N}\left(I_{X}-T\right)\right) \cap\left(\mathcal{N}\left(I_{X}-T\right)\right)^{\perp}$, which coincides wit $\mathcal{R}\left(P\left(I_{X}-T\right)\right)$ by (3.6.5). Hence $\mathcal{R}\left(I_{X}-T\right)+\mathcal{N}\left(I_{X}-T\right)$ is closed.

Finally, we prove that $n^{-2}\left\|T^{n}\right\|_{L(X)} \rightarrow 0$ as $n \rightarrow \infty$. Proceeding by induction, it is easy to check that

$$
T^{n} x=\sum_{k=0}^{\infty} T_{k}^{n} P_{k} x \quad \text { for every } x \in X \text { and } n \in \mathbb{N} .
$$

Furthermore, by induction on $n$ and using (3.6.3), it is not difficult to verify that

$$
\begin{aligned}
& T_{k}^{n}\left(x e_{3 k}+y e_{3 k+1}+z f_{k}\right) \\
& =\left(x-n y \sqrt{1-\cos \theta_{k}}\right) e_{3 k}+y e_{3 k+1}+z\left(1-\sqrt{1-\cos \theta_{k}}\right)^{n} f_{k} \\
& \text { for all } n, k \in \mathbb{N} \text { and }(x, y, z) \in \mathbb{K}^{3} .
\end{aligned}
$$

Now let $n \in \mathbb{N}$. For each $x$ in $X$, let $\left(x_{k}\right)_{k \in \mathbb{N}},\left(y_{k}\right)_{k \in \mathbb{N}},\left(z_{k}\right)_{k \in \mathbb{N}}$ be scalar sequences such that $P_{k} x=x_{k} e_{3 k}+y_{k} e_{3 k+1}+z_{k} f_{k}$ for every $k \in \mathbb{N}$. Then, from (3.6.15) and (3.6.16), we obtain

$$
\left(T^{n}-T^{n+1}\right) x=\sum_{k=0}^{\infty} T_{k}^{n} P_{k} x-\sum_{k=0}^{\infty} T_{k}^{n+1} P_{k} x
$$

$=\sum_{k=0}^{\infty} T_{k}^{n}\left(x_{k} e_{3 k}+y_{k} e_{3 k+1}+z_{k} f_{k}\right)-\sum_{k=0}^{\infty} T_{k}^{n+1}\left(x_{k} e_{3 k}+y_{k} e_{3 k+1}+z_{k} f_{k}\right)$

$=\sum_{k=0}^{\infty}\left(\left(x_{k}-n y_{k} \sqrt{1-\cos \theta_{k}}\right) e_{3 k}+y_{k} e_{3 k+1}+z_{k}\left(1-\sqrt{1-\cos \theta_{k}}\right)^{n} f_{k}\right)$

$$
\begin{aligned}
& -\sum_{k=0}^{\infty}\left(\left(x_{k}-(n+1) y_{k} \sqrt{1-\cos \theta_{k}}\right) e_{3 k}\right. \\
& \left.+y_{k} e_{3 k+1}+z_{k}\left(1-\sqrt{1-\cos \theta_{k}}\right)^{n+1} f_{k}\right) \\
& =\sum_{k=0}^{\infty} \sqrt{1-\cos \theta_{k}}\left(y_{k} e_{3 k}+z_{k}\left(1-\sqrt{1-\cos \theta_{k}}\right)^{n} f_{k}\right) .
\end{aligned}
$$


Since $\left(y_{k} e_{3 k}+z_{k}\left(1-\sqrt{1-\cos \theta_{k}}\right)^{n} f_{k}\right)_{k \in \mathbb{N}}$ is an orthogonal sequence in $X$ and $\left\{e_{3 k}, f_{k}\right\}$ is orthonormal for every $k \in \mathbb{N}$, this gives

$$
\begin{aligned}
\|\left(T^{n}\right. & \left.-T^{n+1}\right) x \|_{X} \\
& =\sqrt{\sum_{k=0}^{\infty}\left(1-\cos \theta_{k}\right)\left\|y_{k} e_{3 k}+z_{k}\left(1-\sqrt{1-\cos \theta_{k}}\right)^{n} f_{k}\right\|_{X}^{2}} \\
& =\sqrt{\sum_{k=0}^{\infty}\left(1-\cos \theta_{k}\right)\left(\left|y_{k}\right|^{2}+\left|z_{k}\right|^{2}\left(1-\sqrt{1-\cos \theta_{k}}\right)^{2 n}\right)} \\
& \leq \sqrt{\sum_{k=0}^{\infty}\left(1-\cos \theta_{k}\right)\left(\left|y_{k}\right|^{2}+\left|z_{k}\right|^{2}\right)} \\
& \leq \sqrt{\sum_{k=0}^{\infty}\left\|x_{k} e_{3 k}+y_{k} e_{3 k+1}+z_{k} f_{k}\right\|_{X}^{2}}=\sqrt{\sum_{k=0}^{\infty}\left\|P_{k} x\right\|_{X}^{2}}=\|x\|_{X}
\end{aligned}
$$

by (3.6.2) and (3.6.4).

We have thus proved that $\left\|T^{n}-T^{n+1}\right\|_{L(X)} \leq 1$ for all $n \in \mathbb{N}$. Hence

$$
\frac{1}{n}\left\|T^{n}-T^{n+1}\right\|_{L(X)} \rightarrow 0 \quad \text { as } n \rightarrow \infty .
$$

From (2.5) we derive that $T$ satisfies condition $\mathcal{E}(2,1)$. Consequently, by Theorem 2.6, we conclude that $n^{-2}\left\|T^{n}\right\|_{L(X)} \rightarrow 0$ as $n \rightarrow \infty$.

Notice that from Theorems 2.8, 3.1 and 3.4 it follows that $\mathcal{R}\left(\left(I_{X}-T\right)^{2}\right)$ is not closed and $\mathcal{R}\left(\left(I_{X}-T\right)^{k}\right)+\mathcal{N}\left(\left(I_{X}-T\right)^{j}\right)$ is closed for no $(k, j) \in$ $\mathbb{Z}_{+} \times \mathbb{N}$ satisfying $k+j>2$.

Let $p \in \mathbb{Z}_{+}, p \geq 3$. We remark that each uniformly $p$-ergodic bounded linear operator $T$ on a Banach space $X$ satisfies the following condition:

$$
\begin{aligned}
& n^{-p} \sum_{k=0}^{n-1} T^{k} \text { converges in } L(X) \text { and } \mathcal{R}\left(\left(I_{X}-T\right)^{p-2}\right)+\mathcal{N}\left(\left(I_{X}-T\right)^{2}\right) \\
& \text { is closed. }
\end{aligned}
$$

Notice that $p-2$ is a positive integer. Also, from (2.9) it follows that for each $T \in L(X)$ satisfying $(3.7), \mathcal{R}\left(\left(I_{X}-T\right)^{j}\right)+\mathcal{N}\left(\left(I_{X}-T\right)^{p-j}\right)$ is closed for all $j=1, \ldots, p-2$.

We are going to prove that condition (3.7) is not necessary for $p$-ergodicity.

We first need a preliminary result. We recall that if $\left(\xi_{n}\right)_{n \in \mathbb{N}}$ is a sequence in a normed space $\mathcal{X}, x \in \mathcal{X}$ and $\left(\tau_{n}\right)_{n \in \mathbb{N}}$ is a strictly increasing sequence of real numbers such that $\lim _{n \rightarrow \infty} \tau_{n}=+\infty$ and $\left(\xi_{n+1}-\xi_{n}\right) /\left(\tau_{n+1}-\tau_{n}\right)$ converges to $x$ in $\mathcal{X}$ as $n \rightarrow \infty$, then also $\tau_{n}^{-1} \xi_{n}$ converges to $x$ in $\mathcal{X}$ as 
$n \rightarrow \infty\left(^{2}\right)$. By applying this with $\tau_{n}=\left(\begin{array}{c}n \\ p+1\end{array}\right)(p \in \mathbb{N})$ and $\xi_{n}=\sum_{k=0}^{n-1} x_{k}$ for a sequence $\left(x_{n}\right)_{n \in \mathbb{N}}$ in a normed space, the following consequence is obtained.

Proposition 3.8. Let $p \in \mathbb{N}, X$ be a normed space, $\left(x_{n}\right)_{n \in \mathbb{N}}$ be a sequence in $X$ and $x \in X$. Then

$$
\lim _{n \rightarrow \infty}\left\|\frac{1}{\left(\begin{array}{l}
n \\
p
\end{array}\right)} x_{n}-x\right\|_{X}=0 \Rightarrow \lim _{n \rightarrow \infty}\left\|\frac{1}{\left(\begin{array}{c}
n \\
p+1
\end{array}\right)} \sum_{k=0}^{n-1} x_{k}-x\right\|_{X}=0 .
$$

Since $\lim _{n \rightarrow \infty}\left(\begin{array}{l}n \\ k\end{array}\right) / n^{k}=1 / k$ ! for every $k \in \mathbb{N}$, Proposition 3.8 yields the following result (which we will use in the next example).

Proposition 3.9. Let $p \in \mathbb{N}, X$ be a normed space, $\left(x_{n}\right)_{n \in \mathbb{N}}$ be a sequence in $X$ and $x \in X$. Then

$$
\lim _{n \rightarrow \infty}\left\|n^{-p} x_{n}-x\right\|_{X}=0 \Rightarrow \lim _{n \rightarrow \infty}\left\|\frac{1}{n^{p+1}} \sum_{k=0}^{n-1} x_{k}-\frac{1}{p+1} x\right\|_{X}=0 .
$$

We take this opportunity to remark that $[\mathrm{Bu} 1,2.4]$ (which is recorded here as Theorem 2.6) can also be derived from Proposition 3.9.

The following example shows that condition (3.7) is not sufficient for uniform $p$-ergodicity. Indeed, we are going to construct a bounded linear operator (on a Hilbert space) which satisfies (3.7) for $p=3$ and nevertheless is not uniformly 3 -ergodic (actually, it is uniformly $p$-ergodic for no $p \in \mathbb{Z}_{+}$).

ExAMPLE 3.10. Let $X$ be an infinite-dimensional separable Hilbert space and $T \in L(X)$ be the operator constructed in Example 3.6. We have proved there that $n^{-2}\left\|T^{n}\right\|_{L(X)} \rightarrow 0$ as $n \rightarrow \infty, \mathcal{R}\left(I_{X}-T\right)+\mathcal{N}\left(I_{X}-T\right)$ is

$\left({ }^{2}\right)$ Although this result is surely known, we have not been able to find any specific bibliographical reference for it in the normed space case (for the scalar case, see for instance [PS, 4.1]). Anyway, it is not difficult to prove. Indeed, for each $\varepsilon>0$, let $\nu_{\varepsilon} \in \mathbb{N}$ be such that $\left\|\left(\xi_{n+1}-\xi_{n}\right) /\left(\tau_{n+1}-\tau_{n}\right)-x\right\|_{\mathcal{X}}<\varepsilon$ and $\tau_{n}>0$ for all $n \in \mathbb{N}$ satisfying $n \geq \nu_{\varepsilon}$. Then, for each $n \in \mathbb{N}$ satisfying $n \geq \nu_{\varepsilon}+1$, we have

$$
\begin{aligned}
\left\|\frac{1}{\tau_{n}} \xi_{n}-x\right\|_{\mathcal{X}} & =\frac{1}{\tau_{n}}\left\|\xi_{\nu_{\varepsilon}}+\sum_{k=\nu_{\varepsilon}}^{n-1}\left(\xi_{k+1}-\xi_{k}\right)-\tau_{n} x\right\|_{\mathcal{X}} \\
& =\frac{1}{\tau_{n}}\left\|\xi_{\nu_{\varepsilon}}-\tau_{\nu_{\varepsilon}} x+\sum_{k=\nu_{\varepsilon}}^{n-1}\left(\tau_{k+1}-\tau_{k}\right)\left(\frac{\xi_{k+1}-\xi_{k}}{\tau_{k+1}-\tau_{k}}\right)-\sum_{k=\nu_{\varepsilon}}^{n-1}\left(\tau_{k+1}-\tau_{k}\right) x\right\|_{\mathcal{X}} \\
& \leq \frac{1}{\tau_{n}}\left\|\xi_{\nu_{\varepsilon}}\right\| \mathcal{X}+\frac{\tau_{\nu_{\varepsilon}}}{\tau_{n}}\|x\|_{\mathcal{X}}+\frac{1}{\tau_{n}} \sum_{k=\nu_{\varepsilon}}^{n-1}\left(\tau_{k+1}-\tau_{k}\right)\left\|\frac{\xi_{k+1}-\xi_{k}}{\tau_{k+1}-\tau_{k}}-x\right\|_{\mathcal{X}} \\
& <\frac{1}{\tau_{n}}\left\|\xi_{\nu_{\varepsilon}}\right\| \mathcal{X}+\frac{\tau_{\nu_{\varepsilon}}}{\tau_{n}}\|x\|_{\mathcal{X}}+\frac{\varepsilon\left(\tau_{n}-\tau_{\nu_{\varepsilon}}\right)}{\tau_{n}} .
\end{aligned}
$$

Hence $\lim \sup _{n \rightarrow \infty}\left\|\tau_{n}^{-1} \xi_{n}-x\right\|_{\mathcal{X}} \leq \varepsilon$, from which the desired result follows. 
closed and nevertheless $T$ is uniformly $p$-ergodic for no $p \in \mathbb{Z}_{+}$. We refer to Example 3.6 also for all the definitions, notations and results concerning $T$ which we do not explicitly recall here.

Let $Q$ denote the orthogonal projection of $X$ onto $\mathcal{N}\left(I_{X}-T\right)$. Notice that $Q=I_{X}-P$. Now let $A$ be the bounded linear operator on the Hilbert space $X \times X$ defined by

$$
A(x, y)=(x-Q y, T y) \quad \text { for every }(x, y) \in X \times X .
$$

We prove that $A$ satisfies condition (3.7) for $p=3$.

We begin by proving that $\mathcal{R}\left(I_{X \times X}-A\right)+\mathcal{N}\left(\left(I_{X \times X}-A\right)^{2}\right)$ is closed. We remark that

(3.10.1) $\left(I_{X \times X}-A\right)(x, y)=\left(Q y,\left(I_{X}-T\right) y\right) \quad$ for every $(x, y) \in X \times X$.

Hence $\mathcal{R}\left(I_{X \times X}-A\right) \subset \mathcal{N}\left(I_{X}-T\right) \times \mathcal{R}\left(I_{X}-T\right)$. We prove that the opposite inclusion also holds. For each $(u, v) \in \mathcal{N}\left(I_{X}-T\right) \times \mathcal{R}\left(I_{X}-T\right)$, there exists $y \in X$ such that $\left(I_{X}-T\right) y=v$. Then

$$
\begin{aligned}
\left(I_{X \times X}-A\right)\left(0_{X}, u+y-Q y\right) & =\left(Q(u+y-Q y),\left(I_{X}-T\right)(u+y-Q y)\right) \\
& =\left(Q u,\left(I_{X}-T\right) y\right)=(u, v),
\end{aligned}
$$

which gives the desired result. We have thus proved that

$$
\mathcal{R}\left(I_{X \times X}-A\right)=\mathcal{N}\left(I_{X}-T\right) \times \mathcal{R}\left(I_{X}-T\right) .
$$

Proceeding by induction, it is not difficult to derive from (3.10.1) that

$$
\begin{aligned}
& \left(I_{X \times X}-A\right)^{n}(x, y)=\left(Q\left(I_{X}-T\right)^{n-1} y,\left(I_{X}-T\right)^{n} y\right) \\
& \text { for all }(x, y) \in X \times X, n \in \mathbb{Z}_{+} .
\end{aligned}
$$

It follows that for each $n \in \mathbb{Z}_{+}$and each $(x, y) \in X \times X$, we have

$$
\begin{aligned}
\left(I_{X \times X}-A\right)^{n}(x, y)=\left(0_{X}, 0_{X}\right) \\
\\
\Leftrightarrow\left\{\begin{array}{l}
Q\left(I_{X}-T\right)^{n-1} y=0_{X} \\
\left(I_{X}-T\right)^{n} y=0_{X}
\end{array}\right. \\
\quad \Leftrightarrow\left(I_{X}-T\right)^{n-1} y \in\left(\mathcal{N}\left(I_{X}-T\right)\right)^{\perp} \cap \mathcal{N}\left(I_{X}-T\right)=\left\{0_{X}\right\} \\
\quad \Leftrightarrow y \in \mathcal{N}\left(\left(I_{X}-T\right)^{n-1}\right) .
\end{aligned}
$$

Hence

(3.10.4) $\mathcal{N}\left(\left(I_{X \times X}-A\right)^{n}\right)=X \times \mathcal{N}\left(\left(I_{X}-T\right)^{n-1}\right) \quad$ for every $n \in \mathbb{Z}_{+}$.

In particular,

$$
\mathcal{N}\left(\left(I_{X \times X}-A\right)^{2}\right)=X \times \mathcal{N}\left(I_{X}-T\right),
$$

which, together with (3.10.2), yields

$$
\begin{aligned}
& \mathcal{R}\left(I_{X \times X}-A\right)+\mathcal{N}\left(\left(I_{X \times X}-A\right)^{2}\right) \subset X \times\left(\mathcal{R}\left(I_{X}-T\right)+\mathcal{N}\left(I_{X}-T\right)\right) \\
& =\left\{0_{X}\right\} \times \mathcal{R}\left(I_{X}-T\right)+X \times \mathcal{N}\left(I_{X}-T\right) \subset \mathcal{R}\left(I_{X \times X}-A\right)+\mathcal{N}\left(\left(I_{X \times X}-A\right)^{2}\right) .
\end{aligned}
$$


Hence

$$
\mathcal{R}\left(I_{X \times X}-A\right)+\mathcal{N}\left(\left(I_{X \times X}-A\right)^{2}\right)=X \times\left(\mathcal{R}\left(I_{X}-T\right)+\mathcal{N}\left(I_{X}-T\right)\right),
$$

which is a closed subspace of $X \times X$, as $\mathcal{R}\left(I_{X}-T\right)+\mathcal{N}\left(I_{X}-T\right)$ is closed in $X$.

Now we prove that $n^{-3} \sum_{k=0}^{n-1} A^{k}$ converges in $L(X \times X)$. Proceeding by induction, it is not difficult to verify that

$$
A^{n}(x, y)=\left(x-\sum_{k=0}^{n-1} Q T^{k} y, T^{n} y\right) \quad \text { for all }(x, y) \in X \times X \text { and } n \in \mathbb{Z}_{+} .
$$

Consequently, for each $n \geq 2$, we have

$$
\begin{aligned}
& \sum_{k=0}^{n-1} A^{k}(x, y) \\
= & (x, y)+\sum_{k=1}^{n-1}\left(x-\sum_{j=0}^{k-1} Q T^{j} y, T^{k} y\right) \\
= & \left(n x-\sum_{k=1}^{n-1} \sum_{j=0}^{k-1} Q T^{j} y, \sum_{k=0}^{n-1} T^{k} y\right) \quad \text { for every }(x, y) \in X \times X .
\end{aligned}
$$

We are going to determine the sequence $\left(\sum_{k=1}^{n-1} \sum_{j=0}^{k-1} Q T^{j}\right)_{n \geq 3}$.

We begin by proving that the sequence $\left(Q T^{n}-Q T^{n+1}\right)_{n \in \mathbb{N}}$ is constant. For each $x \in X$, let $\left(x_{n}\right)_{n \in \mathbb{N}},\left(y_{n}\right)_{n \in \mathbb{N}}$ and $\left(z_{n}\right)_{n \in \mathbb{N}}$ be scalar sequences such that $P_{k} x=x_{k} e_{3 k}+y_{k} e_{3 k+1}+z_{k} f_{k}$ for every $k \in \mathbb{N}$. Since $P_{k}-Q_{k}$ is the orthogonal projection of $X$ onto $\mathcal{N}\left(I_{X_{k}}-T_{k}\right)$ (which is contained in $\left.X_{k}=\operatorname{Span}\left(\left\{e_{3 k}, e_{3 k+1}, f_{k}\right\}\right)\right)$ for every $k \in \mathbb{N}$ and the subspaces $X_{j}$, $j \in \mathbb{N}$, are pairwise orthogonal, from (3.6.4), (3.6.10)-(3.6.12) and (3.6.17) it follows that, for each $n \in \mathbb{N}$,

$$
\begin{aligned}
\left(Q T^{n}-Q T^{n+1}\right) x & =Q \sum_{j=0}^{\infty} \sqrt{1-\cos \theta_{j}}\left(y_{j} e_{3 j}+z_{j}\left(1-\sqrt{1-\cos \theta_{j}}\right)^{n} f_{j}\right) \\
& =\left(I_{X}-P\right) \sum_{j=0}^{\infty} \sqrt{1-\cos \theta_{j}}\left(y_{j} e_{3 j}+z_{j}\left(1-\sqrt{1-\cos \theta_{j}}\right)^{n} f_{j}\right) \\
& =\sum_{k=0}^{\infty}\left(P_{k}-Q_{k}\right) \sum_{j=0}^{\infty} \sqrt{1-\cos \theta_{j}}\left(y_{j} e_{3 j}+z_{j}\left(1-\sqrt{1-\cos \theta_{j}}\right)^{n} f_{j}\right) \\
& =\sum_{k=0}^{\infty} \sqrt{1-\cos \theta_{k}}\left(P_{k}-Q_{k}\right)\left(y_{k} e_{3 k}+z_{k}\left(1-\sqrt{1-\cos \theta_{k}}\right)^{n} f_{k}\right) \\
& =\sum_{k=0}^{\infty} \sqrt{1-\cos \theta_{k}} y_{k} e_{3 k},
\end{aligned}
$$


which is independent of $n$. Hence $Q T^{n}-Q T^{n+1}=Q-Q T$ for all $n \in \mathbb{N}$, which is the desired result. It follows that

$$
Q-Q T^{n}=\sum_{k=0}^{n-1}\left(Q T^{k}-Q T^{k+1}\right)=n(Q-Q T) \quad \text { for every } n \in \mathbb{Z}_{+},
$$

which gives

(3.10.6) $Q T^{n}=n Q T-(n-1) Q=n Q\left(T-I_{X}\right)+Q \quad$ for every $n \in \mathbb{N}$.

By using the well known formula (see for instance [Bu1, (1.9)])

$$
\sum_{j=2}^{k}\left(\begin{array}{l}
j \\
2
\end{array}\right)=\left(\begin{array}{c}
k+1 \\
3
\end{array}\right) \quad \text { for all } k \geq 2
$$

from (3.10.6) we conclude that for $n \geq 3$ we have

$$
\begin{aligned}
\sum_{k=1}^{n-1} \sum_{j=0}^{k-1} Q T^{j} & =\sum_{k=1}^{n-1} \sum_{j=0}^{k-1}\left(j Q\left(T-I_{X}\right)+Q\right) \\
& =\sum_{k=1}^{n-1}\left(\frac{k(k-1)}{2} Q\left(T-I_{X}\right)+k Q\right) \\
& =\left(\sum_{k=2}^{n-1}\left(\begin{array}{l}
k \\
2
\end{array}\right)\right) Q\left(T-I_{X}\right)+\frac{n(n-1)}{2} Q \\
& =\left(\begin{array}{c}
n \\
3
\end{array}\right) Q\left(T-I_{X}\right)+\left(\begin{array}{l}
n \\
2
\end{array}\right) Q .
\end{aligned}
$$

Hence

$$
\left\|\frac{1}{n^{3}} \sum_{k=1}^{n-1} \sum_{j=0}^{k-1} Q T^{j}-\frac{1}{6} Q\left(T-I_{X}\right)\right\|_{L(X)} \rightarrow 0 \quad \text { as } n \rightarrow \infty .
$$

Let $B \in L(X \times X)$ be defined by

$$
B(x, y)=\left(Q\left(T-I_{X}\right) y, 0_{X}\right) \quad \text { for every }(x, y) \in X \times X .
$$

We prove that $n^{-3} \sum_{k=0}^{n-1} A^{k}$ converges to $-\frac{1}{6} B$ in $L(X \times X)$ as $n \rightarrow \infty$. Let $n \geq 2$. From (3.10.5) it follows that for each $(x, y) \in X \times X$ we have

$$
\begin{aligned}
& \left\|\frac{1}{n^{3}} \sum_{k=0}^{n-1} A^{k}(x, y)+\frac{1}{6} B(x, y)\right\|_{X \times X} \\
& \quad=\left\|\left(\frac{1}{n^{2}} x-\frac{1}{n^{3}} \sum_{k=1}^{n-1} \sum_{j=0}^{k-1} Q T^{j} y+\frac{1}{6} Q\left(T-I_{X}\right) y, \frac{1}{n^{3}} \sum_{k=0}^{n-1} T^{k} y\right)\right\|_{X \times X}
\end{aligned}
$$




$$
\begin{aligned}
& \leq \frac{1}{n^{2}}\|x\|_{X}+\left\|\frac{1}{n^{3}} \sum_{k=1}^{n-1} \sum_{j=0}^{k-1} Q T^{j}-\frac{1}{6} Q\left(T-I_{X}\right)\right\|_{L(X)}\|y\|_{X} \\
& \quad+\frac{1}{n^{3}}\left\|\sum_{k=0}^{n-1} T^{k}\right\|_{L(X)}\|y\|_{X} \\
& \leq\left(\frac{1}{n^{2}}+\left\|\frac{1}{n^{3}} \sum_{k=1}^{n-1} \sum_{j=0}^{k-1} Q T^{j}-\frac{1}{6} Q\left(T-I_{X}\right)\right\|_{L(X)}\right. \\
& \left.\quad+\frac{1}{n^{3}}\left\|\sum_{k=0}^{n-1} T^{k}\right\|_{L(X)}\right)\|(x, y)\|_{X \times X} .
\end{aligned}
$$

Hence

$$
\begin{aligned}
& \left\|\frac{1}{n^{3}} \sum_{k=0}^{n-1} A^{k}+\frac{1}{6} B\right\|_{L(X \times X)} \\
& \leq \frac{1}{n^{2}}+\left\|\frac{1}{n^{3}} \sum_{k=1}^{n-1} \sum_{j=0}^{k-1} Q T^{j}-\frac{1}{6} Q\left(T-I_{X}\right)\right\|_{L(X)}+\frac{1}{n^{3}}\left\|\sum_{k=0}^{n-1} T^{k}\right\|_{L(X)}
\end{aligned}
$$

for $n \geq 2$. Since $\lim _{n \rightarrow \infty} n^{-2}\left\|T^{n}\right\|_{L(X)}=0$, from Proposition 3.9 it follows that

$$
\lim _{n \rightarrow \infty} \frac{1}{n^{3}}\left\|\sum_{k=0}^{n-1} T^{k}\right\|_{L(X)}=0 .
$$

This, together with (3.10.7) and (3.10.8), yields

$$
\lim _{n \rightarrow \infty}\left\|\frac{1}{n^{3}} \sum_{k=0}^{n-1} A^{k}+\frac{1}{6} B\right\|_{L(X \times X)}=0,
$$

which is the desired result.

We have thus proved that $A$ satisfies condition (3.7) for $p=3$.

Finally, we prove that $A$ is uniformly $p$-ergodic for no $p \in \mathbb{Z}_{+}$. We begin by remarking that $(3.10 .3)$ yields

$$
\mathcal{R}\left(\left(I_{X \times X}-A\right)^{n}\right) \subset X \times \mathcal{R}\left(\left(I_{X}-T\right)^{n}\right) \quad \text { for all } n \in \mathbb{Z}_{+} \cdot
$$

Since $n^{-2}\left\|T^{n}\right\|_{L(X)} \rightarrow 0$ as $n \rightarrow \infty$ and $T$ is not uniformly 2-ergodic, from Theorems 2.8 and 3.1 it follows that $\delta\left(I_{X}-T\right)=\infty$. Hence, for each $n \in \mathbb{Z}_{+}$, there exists $y_{n} \in X$ such that $\left(I_{X}-T\right)^{n} y_{n} \notin \mathcal{R}\left(\left(I_{X}-T\right)^{n+1}\right)$, which, by (3.10.3) and (3.10.9), gives

$$
\begin{aligned}
\left(Q\left(I_{X}-T\right)^{n-1} y_{n},\left(I_{X}\right.\right. & \left.-T)^{n} y_{n}\right) \\
& \in \mathcal{R}\left(\left(I_{X \times X}-A\right)^{n}\right) \backslash\left(X \times \mathcal{R}\left(\left(I_{X}-T\right)^{n+1}\right)\right) \\
& \subset \mathcal{R}\left(\left(I_{X \times X}-A\right)^{n}\right) \backslash \mathcal{R}\left(\left(I_{X \times X}-A\right)^{n+1}\right) .
\end{aligned}
$$


We have thus proved that $\delta\left(I_{X \times X}-A\right)=\infty$. Consequently, by Theorems 2.8 and $3.1, A$ is uniformly $p$-ergodic for no $p \in \mathbb{Z}_{+}$. In particular, $A$ is not uniformly 3-ergodic.

We remark that since $A$ is not uniformly 3 -ergodic and $n^{-3} \sum_{k=0}^{n-1} A^{k}$ converges in $L(X \times X)$, it follows that $\mathcal{R}\left(\left(I_{X \times X}-A\right)^{2}\right)+\mathcal{N}\left(I_{X \times X}-A\right)$ is not closed. Consequently, by $(2.9), \mathcal{R}\left(\left(I_{X \times X}-A\right)^{3}\right)$ is not closed. Furthermore, by Theorem 3.4, $\mathcal{R}\left(\left(I_{X \times X}-A\right)^{k}\right)+\mathcal{N}\left(\left(I_{X \times X}-A\right)^{j}\right)$ is closed for no $(k, j) \in$ $\mathbb{Z}_{+} \times \mathbb{N}$ satisfying $k+j>3$.

\section{References}

[Be] M. Berkani, Restriction of an operator to the range of its powers, Studia Math. 140 (2000), 163-175.

[Bu1] L. Burlando, A generalization of the uniform ergodic theorem to poles of arbitrary order, ibid. 122 (1997), 75-98.

[Bu2] -, On nilpotent operators, ibid., to appear.

[D1] N. Dunford, Spectral theory. I. Convergence to projections, Trans. Amer. Math. Soc. 54 (1943), 185-217.

[D2] - Spectral theory, Bull. Amer. Math. Soc. 49 (1943), 637-651.

[GZ] S. Grabiner and J. Zemánek, Ascent, descent, and ergodic properties of linear operators, J. Operator Theory 48 (2002), 69-81.

[HP] E. Hille and R. S. Phillips, Functional Analysis and Semi-Groups, Amer. Math. Soc., 1957.

[K] T. Kato, Perturbation theory for nullity, deficiency and other quantities of linear operators, J. Anal. Math. 6 (1958), 261-322.

[KMMP] J. J. Koliha, M. Mbekhta, V. Müller and P. W. Poon, Corrigendum and addendum: "On the axiomatic theory of spectrum II", Studia Math. 130 (1998), 193-198.

[Lab] J. P. Labrousse, Les opérateurs quasi-Fredholm: une généralisation des opérateurs semi-Fredholm, Rend. Circ. Mat. Palermo (2) 29 (1980), 161-258.

[LM $\quad$ K. B. Laursen and M. Mbekhta, Operators with finite chain length and the ergodic theorem, Proc. Amer. Math. Soc. 123 (1995), 3443-3448.

[Lay] D. C. Lay, Spectral analysis using ascent, descent, nullity and defect, Math. Ann. 184 (1970), 197-214.

[Li] M. Lin, On the uniform ergodic theorem, Proc. Amer. Math. Soc. 43 (1974), 337-340.

[MM] M. Mbekhta and V. Müller, On the axiomatic theory of spectrum II, Studia Math. 119 (1996), 129-147.

[MO] M. Mbekhta et A. Ouahab, Perturbation des opérateurs s-réguliers, in: Topics in Operator Theory, Operator Algebras and Applications (Timişoara, 1994), A. Gheondea et al. (eds.), Institute of Mathematics of the Romanian Academy, $1995,239-249$.

[MZ] M. Mbekhta et J. Zemánek, Sur le théorème ergodique uniforme et le spectre, C. R. Acad. Sci. Paris Sér. I Math. 317 (1993), 1155-1158.

[P] P. W. Poon, The stability radius of a quasi-Fredholm operator, Proc. Amer. Math. Soc. 126 (1998), 1071-1080. 
[PS] R. E. Powell and S. M. Shah, Summability Theory and its Applications, Van Nostrand Reinhold, 1972.

[S] H. H. Schaefer, Topological Vector Spaces, Macmillan, 1966.

[TL] A. E. Taylor and D. C. Lay, Introduction to Functional Analysis, 2nd ed., Wiley, 1980.

[W] H.-D. Wacker, Über die Verallgemeinerung eines Ergodensatzes von Dunford, Arch. Math. (Basel) 44 (1985), 539-546.

Dipartimento di Matematica dell'Università di Genova

Via Dodecaneso 35

16146 Genova, Italy

E-mail: burlando@dima.unige.it

Received September 29, 2003

Revised version November 7, 2003 